\title{
Charge States in Andreev Quantum Dots
}

\author{
Ivan A. Sadovskyy, Gordey B. Lesovik and \\ Valerii M. Vinokur \\ Additional information is available at the end of the chapter \\ http://dx.doi.org/10.5772/60482
}

\begin{abstract}
We study the charge states in Andreev quantum dot, the metallic quantum dot coupled to the superconducting ring. We show that breaking the electron-hole symmetry in a superconductor generates non-integer charge, localized in a metallic part of the Andreev quantum dot. We demonstrate that this non-integer charge varies continuously as a result of the electrostatic gating and/or change in the phase difference between the superconducting banks. We investigate charge fluctuations associated with the electron-phonon coupling and Coulomb interactions. We propose a recipe for measuring the charge enabling the design of a sensor for weak magnetic fields whose working element is the Andreev quantum dot.
\end{abstract}

Keywords: superconductivity, Andreev quantum dot, non-integer charge

\section{Introduction}

A relation between the charge and phase of the order parameter in superconductors is one of the central issues of physics of superconductivity. The nondissipative supercurrent is related to the gradient of the superconducting phase, in particular, Josephson current is caused by the phase difference $\varphi$ at the banks of the contacts $[1,2]$. The static charges are intimately connected with the singularities in the phase distribution. The point singularity associated with the zero of the order parameter, around which the phase, when encircling singularity, gains $2 \pi$ are superconducting vortices [3], which can be viewed as filaments of the normal metal (vortex normal cores) surrounded by encircling supercurrents. The quasiparticle states that form in the core, so-called Caroli-de Gennes-Matricon or Andreev states, carry the small excess charge $e k_{\mathrm{F}}$ per unite length $[4,5]$, where $e$ is the electronic charge and $k_{\mathrm{F}}$ is the Fermi wave vector of the underlying normal metal. This excessive charge stems from the violation of symmetry between electrons and holes. Since Andreev state are 
the base for variety of the emerging superconducting devices, like, for example, Josephson transistors [6-8], the study of physics of the charge states associated with the Andreev levels is of prime importance not only from the fundamental science viewpoint but is critical for applications of superconductors.

We will focus on the charge states associated with the Andreev levels in a so-called Andreev quantum dot. The latter is a metallic quantum dot coupled via tunneling contacts to a superconducting ring $[9,10]$, see Fig. 1 , where breaking the symmetry between electrons and holes gives rise to a localized charge $[10,11]$. This charge can be continuously changed by variation of the phase difference between the superconducting banks $\varphi$ and the gate voltage $V_{\mathrm{g}}$, hence the charge is not necessarily an integer. In particular, a singly excited state (where the number of Bogoliubov quasiparticles is odd) carries an integer charge, whereas the ground state and a doubly excited states (with the even number of Bogoliubov quasiparticles) carry non-integer charge.

Condensed matter physics is mostly dealing with the situations where the charges appear as multiple integers of the electron elemental charge $e$. Notable examples where fractional charges appear are fractional Hall effect [12-14] and Luttinger liquid [15-17]. In either case fractional charge appears due to averaging over time. The charges at the Andreev dot that we will discuss here also appear due to averaging over time, but do not have some fixed fractionality and can be altered continuously by tuning external parameters such as superconducting phase difference and gate voltage. Continuously tunable charges in mesoscopic systems were discussed, e.g., in Refs. [18, 19]. There, the appearance of the non-integer charge is ensured by the peculiarity of corresponding wave function partially localized in the quantum dot; exponentially decaying wave function "tails" comprise the rest of an integer charge. Here we will discuss the non-integer charge completely localized in a quantum dot, while the rest is localized far away in the superconducting condensate. Non-integer charges we will be dealing with, resemble fractional charges associated with the excitations in superconductors and are discussed in the context of the charge relaxation in nonequilibrium superconductivity [20,21]. The examples of measurable manifestations of the non-integer charge include the telegraph signal arising due to stochastic occupation of the Andreev levels $[9,10]$ and the dependence of the charge upon magnetic flux piercing a superconducting ring. This opens route for designing the Andreev dot-based device for measuring weak magnetic fields [22].

This chapter is organized as follows: In Sec. 2 we introduce preliminaries relating to the origin of the fractional charge in the Andreev quantum dot, and in Secs. 3 and 4 turn to the detailed analysis of the charge and energy states respectively. In Secs. 5-7 we describe the

(a)

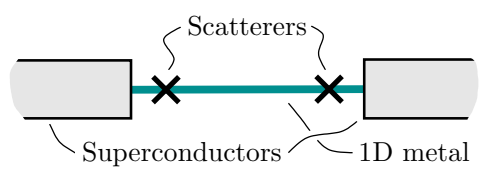

(b)

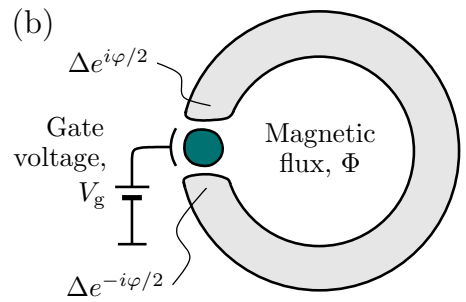

Figure 1. (a) Sketch of the Andreev quantum dot: A quantum dot connected to superconducting banks. (b) Andreev quantum dot driving by magnetic flux $\Phi=(\varphi / 2 \pi) \Phi_{0}$ threading the superconducting loop and by gate voltage $V_{\mathrm{g}}$. 
possible sources of the fluctuations of the fractional charge. Section 8 is devoted to effects of Coulomb interaction. In Sec. 9 we discuss the scalability of the charge. In Sec. 10 we describe the means for detecting the fractional charge, and in Sec. 11 the possibility of using the fractional charge in the Andreev quantum dot for detecting weak magnetic fields.

\section{The origin of the fractional charge}

Andreev reflection is at the heart of the physics of mesoscopic superconducting structures [23-25]. At the normal metal-superconductor (NS) interface an electron (hole) incident on the interface from the normal metal $(\mathrm{N})$ at energies $E$ less than the superconducting energy gap, $\left|E-E_{\mathrm{F}}\right|<\Delta\left(E_{\mathrm{F}}\right.$ and $\Delta$ are the Fermi energy and the superconducting gap, respectively), cannot enter the superconductor $(S)$ and is reflected as a hole (electron) moving in the direction opposite to that of incident electron (hole) into the normal metal. To form a hole, the electron in the $|k\rangle$ state pulls an electron in the $|-k\rangle$ state and transmits into the superconductor forming a Cooper pair. Note that at the ideal NS-interface the reflection of an electron is complete (i.e. the reflection coefficient is unity) and does not depend on the energy of the incident electron.

In the superconductor-normal metal-superconductor (SNS) contact, an electron impinging on one of the interfaces is Andreev reflected and converted into a hole moving in the opposite direction, thus generating a Cooper pair at the interface, and is converted back to an electron, leading to the destruction of the Cooper pair in the other superconductor, see Fig. 2(a). As a result of this cycle, a pair of correlated electrons is transferred from one superconductor to another, creating a supercurrent flow across the junction. The resulting state in a normal region maintains the electron-hole symmetry hence carrying an integer, if measured in the electron charge units, charge (we disregard hereafter a slight violation of the electron-hole symmetry of the order of $\Delta / E_{\mathrm{F}}$ arising due to finiteness of the Fermi energy).

Adding a normal scatterer to each of the NS-interfaces would form so-called non-ideal normal metal-insulator-superconductor (NIS) interface, at which the scattering acquires both, Andreev and normal components, the latter being strongly energy-dependent, see Fig. 2(b). As a result in an SINIS junction the electron-hole symmetry breaks down. This is easily understood in terms of electron- and hole lifetimes within the normal region. In SNS contact these times coincide with the accuracy of $\Delta / E_{\mathrm{F}}$. The presence of scattering resonances breaks this symmetry drastically. Indeed, if the quasiparticle energy, $\varepsilon$, is close to resonance $\varepsilon_{\mathrm{D}}$ (both
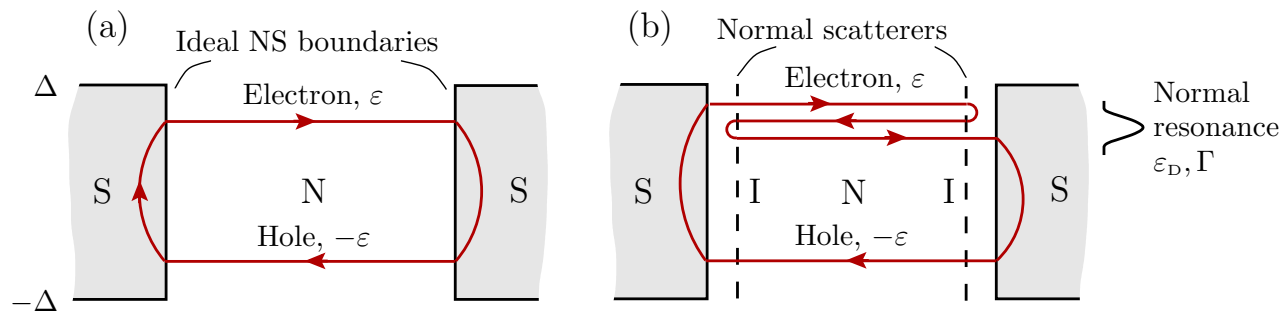

Figure 2. (a) Andreev reflection in the SNS junction with the ideal NS interfaces. (b) Andreev reflection in the SINIS junction: the scattering at the INI part is of the resonance character which results in violation of the electron-hole symmetry. The panel displays electron-like resonance, $\varepsilon_{\mathrm{D}}>0$. 
$\varepsilon$ and $\varepsilon_{\mathrm{D}}$ are measured from the Fermi level), more precisely, if $\left|\varepsilon-\varepsilon_{\mathrm{D}}\right|<\Gamma$, where $\Gamma$ is the resonance half-width, the probability of the normal reflection is appreciably enhanced, hence so does the time the quasiparticle dwells near the resonance. Shown in Fig. 2(b) is the electron-like resonance with $\varepsilon_{\mathrm{D}}>0$ where a quasiparticle spends the better share of time in the electron-like rather than in the hole-like state. Inversely, for $\varepsilon_{\mathrm{D}}<0$, the quasiparticle is predominantly a hole-like one.

More insight into Andreev states in a SINIS contact can be gained via the initial study a corresponding NININ structure where all the superconductors are substitute by normal metals. In this case every resonance turns into the corresponding Andreev level. Electron-like resonances above Fermi level, i.e. with $\varepsilon>0$, correspond to electron-like Andreev states with the negative excitation charge, while hole-like resonances with $\varepsilon<0$ correspond to hole-like Andreev levels with the positive excitation charge. These states can transform one into another via changing the gate potential $V_{\mathrm{g}}$ or the phase difference $\varphi$ along the contact.

\section{A single Andreev level}

In this section we discuss a single quantum conducting channel for a spinless particle, i.e. a single Andreev level. We demonstrate that such a channel can carry a charge $Q \in[0 \ldots 2 e]$. Hereafter $e$ is the negative charge of the electron, accordingly, $-e$ is the positive charge of the hole. We focus on the study of the charge $Q$ as a function of the difference of the superconducting phase $\varphi$, and the position, $\varepsilon_{\mathrm{D}}$, of the normal resonance with respect to the Fermi level. We thus adopt a model where both superconductor and metal are one-dimensional (1D), which captures all the essentials of SINIS behavior. Accounting for realistic peculiarity of the massive superconductor/normal metal contact would change quantitative characteristics of transport across SINIS, but not its qualitative behavior.

In order for only a single Andreev level contributed to a current, only a single resonance must fall into the superconducting gap energy interval, $\varepsilon_{\mathrm{D}} \in[-\Delta \ldots \Delta]$, and accordingly the resonances spacing, $\delta$, well exceeded the superconducting gap,

$$
\delta \gg \Delta
$$

This condition is equivalent to the requirement that the SINIS junction were short, i.e. that $L \ll \xi$, where $L$ is the length of the normal segment, and $\xi=\hbar v_{\mathrm{F}} / \Delta$ is the coherence length. Furthermore, the "tails" of the adjacent resonances should not appear within the $[-\Delta \ldots \Delta]$ interval, therefore, we let also the half-widths of the adjacent resonances which are approximately the same as that of the resonance involved, $\Gamma$, were much less the resonance spacing, $\Gamma \ll \delta$. All these conditions are easily realized in experiment, see Fig. 3 .

The Andreev quantum dots can be designed on the basis of single-wall carbon nanotubes [2630] or molecule [31-33]. The nanotube can be attached suspending between the two superconducting leads, or else can be placed on the dielectric substrate, the superconducting contacts being sputtered on it. The normal scatterers can be realized via two extra gates that suppress electron density at the predesigned points creating two 1D scatterers with the transmission amplitudes $t_{\mathrm{L}}$ and $t_{\mathrm{R}}$, see Fig. 3 . The gates form the Andreev quantum dot at the nanotube. The main gate of the length of the order of the normal part of the junction creates 
an extra potential $V_{\mathrm{g}}$ in the nanotube which tunes the position of the normal resonance with respect to the Fermi energy $\varepsilon_{\mathrm{D}}$.

We start our description of the charge states in SINIS adopting the characteristics (positions and half-widths) of the resonances in the NININ junction, equivalent to the SINIS one. To this end let us consider the problem of the eigenvalues of the normal state Hamiltonian $\hat{\mathcal{H}}_{0} \Psi=E \Psi$, defined by

$$
\hat{\mathcal{H}}_{0}=-\frac{\hbar^{2}}{2 m} \frac{\partial^{2}}{\partial x^{2}}+U(x)-E_{\mathrm{F}} .
$$

The 1D potential describing the NININ junction,

$$
\left.U(x)=U_{\mathrm{ps}, \mathrm{L}}(x+L / 2)+U_{\mathrm{ps}, \mathrm{R}}(x-L / 2)+e V_{\mathrm{g}} \theta(L / 2-|x|)\right],
$$

consists of two contributions, $U_{\mathrm{ps, \textrm {L }}}$ and $U_{\mathrm{ps}, \mathrm{R}}$, from the point scatterers and of the gate potential $V_{\mathrm{g}}$, which is taken to be much smaller than the Fermi energy, $e V_{\mathrm{g}} \ll E_{\mathrm{F}}$. The scatterers are endowed with the transmission and reflection amplitudes $t_{\ell}=\sqrt{T_{\ell}} e^{\chi_{\ell}^{t}}$ and $\sqrt{R_{\ell}} e^{\chi_{\ell}^{r}}$, respectively; $\ell=\mathrm{L}$ (left), $\mathrm{R}$ (right), $R_{\ell}+T_{\ell}=1$.

In Eq. (3) the Heaviside step function is $\theta(x)=0$ for $x<0$ and $\theta(x)=1$ for $x>0$. The eigenvalues of this problem are resonance energies

$$
E_{n}=\varepsilon_{L}\left(n \pi-\frac{\chi_{\mathrm{L}}^{r}}{2}-\frac{\chi_{\mathrm{R}}^{r}}{2}\right)^{2}
$$

where $\varepsilon_{L}=\hbar^{2} / 2 m L^{2}$ is the energy defining the quantization along the contact. The levels (4) separation (spacing) is

$$
\delta_{n}=\frac{E_{n+1}-E_{n-1}}{2}=2 \pi \sqrt{\varepsilon_{L} E_{n}} \approx \frac{2 E_{n}}{n}
$$

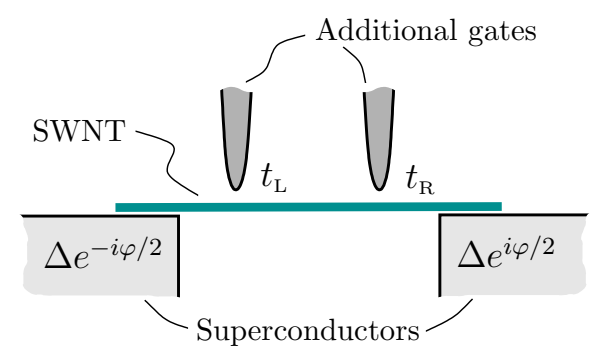

Figure 3. The Andreev quantum dot can be realized adding two gates to the SINIS structure, which deplete the electron density at the designed spots and form the effective barriers with the transmission amplitudes $t_{\mathrm{L}}$ and $t_{\mathrm{R}}$. 
and have half-widths

$$
\Gamma_{n}=\frac{1-\sqrt{R_{\mathrm{L}} R_{\mathrm{R}}}}{\sqrt[4]{R_{\mathrm{L}} R_{\mathrm{R}}}} \sqrt{\varepsilon_{L} E_{n}} .
$$

If $T_{\mathrm{L}}, T_{\mathrm{R}} \ll 1$ formula (6) reduces to

$$
\Gamma_{n}=\frac{T_{\mathrm{L}}+T_{\mathrm{R}}}{2} \sqrt{\varepsilon_{L} E_{n}}=\frac{T_{\mathrm{L}}+T_{\mathrm{R}}}{2} \frac{\delta_{n}}{2 \pi}
$$

Gate potential shifts all the resonances over $e V_{\mathrm{g}}$. Let us denote the position of the $n$-th resonance with respect to Fermi level $E_{\mathrm{F}}$ as $\varepsilon_{\mathrm{D}}=E_{n}+e V_{\mathrm{g}}-E_{\mathrm{F}}$. Hereafter, the subscript $n$ enumerating the resonances we will be omitted.

Now let us return from the ordinary normal quantum dot to the Andreev one, replacing the normal banks by the superconducting ones. To include the Andreev reflection processes in the SINIS, we solve Bogoliubov-de Gennes equations choosing the states with $\varepsilon_{\mathrm{A}} \geqslant 0$,

$$
\left[\begin{array}{cc}
\hat{\mathcal{H}}_{0}(x) & \hat{\Delta}(x) \\
\hat{\Delta}^{*}(x) & -\hat{\mathcal{H}}_{0}(x)
\end{array}\right]\left[\begin{array}{l}
u(x) \\
v(x)
\end{array}\right]=\varepsilon_{\mathrm{A}}\left[\begin{array}{l}
u(x) \\
v(x)
\end{array}\right]
$$

with the piecewise smooth superconducting gap

$$
\hat{\Delta}(x)=\Delta\left[\theta(-x-L / 2) e^{-i \varphi / 2}+\theta(x-L / 2) e^{i \varphi / 2}\right]
$$

Here $u(x)$ and $v(x)$ are the electron- and hole components of the wave function and $\varepsilon_{\mathrm{A}}$ is an excitation energy for the system, i.e. the energy acquired by the system upon adding a quasiparticle. The superconducting gap $\hat{\Delta}(x)$ describes bulk superconductors having the phase $-\varphi / 2$ at the left bank and $\varphi / 2$ at the right hand side one.

One can solve Eqs. (8) by matching plane wave solution for the normal and decaying solutions in a superconductor. We employ, however a more technique approach based on the scattering matrices approach, which utilizes the fact that the transfer-matrices of the series of the contacts is merely a product of all the respective sequential matrices corresponding to individual contact [34]. This is in fact a generalization of the wave functions matching approach but applied to NS boundaries only and including the characteristics of the normal part as parameters. Let us denote the amplitudes of transmission and reflection of the INI part as $\sqrt{T} e^{\chi^{t}}$ and $\sqrt{R} e^{\chi^{r}}$, respectively. In the Andreev approximation we find

$$
\cos \left(S_{+}-S_{-}-2 \alpha\right)=\sqrt{R_{+} R_{-}} \cos \beta+\sqrt{T_{+} T_{-}} \cos \varphi
$$

Here subscripts " \pm " refer to the probabilities and phases of reflection/transmission from/through the INI part corresponding to energies $\pm \varepsilon_{\mathrm{A}}$. This parametrization presumes no electron-hole scattering within INI. The phase $\alpha=\arccos \left(\varepsilon_{\mathrm{A}} / \Delta\right)$ is but the Andreev reflection phase at the ideal NS boundary at $\varphi=0$. The phases $S_{ \pm}=\chi_{ \pm}^{t}+k_{\mathrm{e}} / \mathrm{h} L$ stand for the phase 


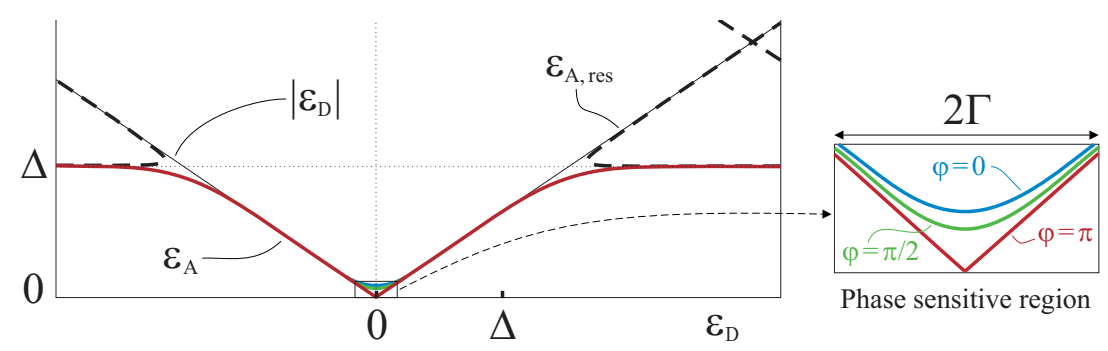

Figure 4. Andreev energy $\varepsilon_{\mathrm{A}}$ (thick solid line) and Andreev resonances $\varepsilon_{\mathrm{A} \text {,res }}$ (dashed line) as functions of the position of the normal resonance $\varepsilon_{\mathrm{D}}$. There are regions where both $\varepsilon_{\mathrm{A}}$ and $\varepsilon_{\mathrm{A} \text {,res }}$ follow the behaviors of the normal resonances $\left|\varepsilon_{\mathrm{D}}\right|$ shown by thin solid lines, holewise, with $\varepsilon_{\mathrm{D}}<0$ and electronwise with $\varepsilon_{\mathrm{D}}>0$ slopes. The inset on the right shows the magnified phase-sensitive region. When finding numerical solutions illustrated by the figure $\Gamma=0.1 \Delta$ was used and symmetric contact with $t_{\mathrm{L}}=t_{\mathrm{R}}$ was chosen.

gain of electron and holes in the normal region, where $k_{\mathrm{e} / \mathrm{h}}=\sqrt{2 m\left(E_{\mathrm{F}} \pm \varepsilon_{\mathrm{A}}\right)} / \hbar$ are the respective wave vectors. For symmetric barriers the phase $\beta=\left(\chi_{+}^{t}-\chi_{+}^{r}\right)-\left(\chi_{-}^{t}-\chi_{-}^{r}\right)$ and an integer multiple of $\pi$ and generates a smooth function $\sqrt{R_{+} R_{-}} \cos \beta$, changing its sign at each resonance $[8,35]$.

In the case of the SINIS contact, Eq. (10) acquires a form:

$$
\begin{aligned}
\left(R_{\mathrm{L}}+R_{\mathrm{R}}\right) \cos \left(2 \pi \frac{I \varepsilon_{\mathrm{A}}}{\delta}\right)-4 \sqrt{R_{\mathrm{L}} R_{\mathrm{R}}} \cos ( & \left.2 \pi \frac{\varepsilon_{\mathrm{D}}}{\delta}\right) \sin ^{2} \alpha+T_{\mathrm{L}} T_{\mathrm{R}} \cos \varphi \\
& =\cos \left(2 \alpha-2 \pi \frac{\varepsilon_{\mathrm{A}}}{\delta}\right)+R_{\mathrm{L}} R_{\mathrm{R}} \cos \left(2 \alpha+2 \pi \frac{\varepsilon_{\mathrm{A}}}{\delta}\right) .
\end{aligned}
$$

The dependence of the Andreev energy $\varepsilon_{\mathrm{A}}$ upon the difference of the superconducting phases $\varphi$ and the position of the normal resonance (i.e. upon the gate voltage) $\varepsilon_{\mathrm{D}}$ is found numerically and shown in Fig. 4.

One can analyze Eq. (11) in the following interesting situations. First, if the quantum dot is far from the resonance conditions, $\left|\varepsilon_{\mathrm{D}}\right| \gg \Delta$, the Andreev energy is given by the expression

$$
\varepsilon_{\mathrm{A}} \approx \Delta\left(1-\frac{\Gamma^{2}}{2 \varepsilon_{\mathrm{D}}^{2}}\right)
$$

Second, if the resonance falls within the superconducting gap but is apart from the Fermi energy over than $\Gamma$, i.e. if $\Gamma \lesssim\left|\varepsilon_{\mathrm{D}}\right| \lesssim \Delta$ ), the Andreev energy depends linearly on the position of the normal resonance:

$$
\varepsilon_{\mathrm{A}} \approx\left(1-\frac{\Gamma}{\Delta}\right)\left|\varepsilon_{\mathrm{D}}\right|
$$


Finally, if the resonance approaches to the Fermi level so that $\left|\varepsilon_{D}\right| \lesssim \Gamma$ and $\Gamma \ll \Delta$, the Andreev reflection grows sensitive to the superconducting phase difference $\varphi$ and is given by

$$
\varepsilon_{\mathrm{A}}=\left(1-\frac{\Gamma}{\Delta}\right) \sqrt{\varepsilon_{\mathrm{D}}^{2}+\tilde{\Gamma}^{2}}
$$

where

$$
\tilde{\Gamma}=\Gamma \sqrt{\cos ^{2} \frac{\varphi}{2}+A^{2}}, \quad A=\frac{\left|T_{\mathrm{L}}-T_{\mathrm{R}}\right|}{2 \sqrt{T_{\mathrm{L}} T_{\mathrm{R}}}} .
$$

The latter case (often referred to as an infinite gap limit) is especially interesting to us as containing measurable $\varepsilon_{\mathrm{A}}(\varphi)$ dependence. Restricting ourselves to the main order in $\Gamma / \Delta$, we rewrite formula (14) as

$$
\varepsilon_{\mathrm{A}}=\sqrt{\varepsilon_{\mathrm{D}}^{2}+\tilde{\Gamma}^{2}}
$$

In this limit both wave function components, $u(x)$ and $v(x)$, are different from zero solely in the normal region

$$
\left[\begin{array}{l}
u(x) \\
v(x)
\end{array}\right]=\left\{\begin{array}{cl}
0, & |x|>L / 2, \\
{\left[\begin{array}{cl}
C_{\mathrm{e}} \rightarrow e^{i k_{\mathrm{e}} x}+C_{\mathrm{e}}^{\leftarrow} e^{-i k_{\mathrm{e}} x} \\
C_{\mathrm{h}}^{\leftarrow} e^{i k_{\mathrm{h}} x}+C_{\mathrm{h}}^{\rightarrow} e^{-i k_{\mathrm{h}} x}
\end{array}\right],|x|<L / 2 .}
\end{array}\right.
$$

with the coefficients governing the relative contributions from the electronwise and holewise being

$$
\mathrm{C}_{\mathrm{e} / \mathrm{h}}^{\rightarrow}=C_{\mathrm{e} / \mathrm{h}}^{\leftarrow}=\sqrt{\left(1 \pm \varepsilon_{\mathrm{D}} / \varepsilon_{\mathrm{A}}\right) / 2 L}
$$

Let us discuss at some more length on the conditions under which Eqs. (16) and (17) hold. We need in fact two of them: (i) A large superconducting gap so that $\Delta \gg \Gamma$, which allows for disregarding the continuous spectrum and (ii) Small, as compared to the superconducting coherence length, the length of the contact, $L \ll \xi$. Note here that under these conditions the Andreev energy $\varepsilon_{\mathrm{A}}$ depends strongly upon $\varphi$ in the window $\left|\varepsilon_{\mathrm{D}}\right| \lesssim \Gamma$. Writing down these conditions via the parameters ordinarily relating to the coherence length $h \xi=\hbar v_{\mathrm{F}} / \Delta$ and the transparency of one of the barriers, one finds for the conditions (i) and (ii), respectively:

$$
\begin{aligned}
& \frac{\Gamma}{\Delta}=T_{1} \frac{\hbar v_{\mathrm{F}}}{L \Delta} \ll 1, \\
& \frac{L}{\xi}=\frac{L \Delta}{\hbar v_{\mathrm{F}}} \ll 1 .
\end{aligned}
$$

We have taken a symmetric SINIS contact for the sake of simplicity. Both conditions (19) and (20) are satisfied simultaneously if we take e.g. $L / \xi=0.1$ and $T=0.01$. One sees immediately why one can neglect the wave function within the superconductors, see Eq. (17): the size of the region where the wave function dies out fast grows with $\xi / L$, whereas the amplitude square is proportional to $\Gamma / \Delta$. Hence integrating the square of the wave function 


$$
\begin{aligned}
& |2\rangle \text {, singlet } \\
& |1\rangle \text {, doublet } \\
& |0\rangle \text {, singlet }
\end{aligned}
$$

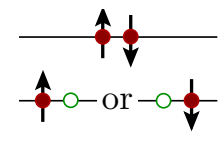

$E_{2}=U_{0}+2 \varepsilon_{\mathrm{A}}$

$E_{1}=U_{0}+\varepsilon_{\mathrm{A}}$

$E_{0}=U_{0}$

Figure 5. Classification of the energy levels in the SINIS junction.

over the length is proportional to $\xi$, one gets a quantity which is well less than that coming out from integrating over the normal region of smaller length.

In the limit of the infinite superconducting gap, we can neglect by the continuous spectrum and take into account only four states. The ground state is the quasiparticle-free state $|0\rangle$ with the energy

$$
E_{0}=U_{0},
$$

where $U_{0}$ is the $c$-number factor in the Bogoliubov transformation. We count all the energies from the Fermi energy $E_{\mathrm{F}}$. The first excited state is $|1\rangle$ with one Bogoliubov particle is twice degenerate with respect to the spin (in order to discriminate different spin states we will be using notations $\left.|\uparrow\rangle=\hat{a}_{\uparrow}^{\dagger}|0\rangle,|\downarrow\rangle=\hat{a}_{\downarrow}^{\dagger}|0\rangle\right)$. Its energy is

$$
E_{1}=U_{0}+\varepsilon_{\mathrm{A}}
$$

which is obtained by adding the excitation energy $\varepsilon_{\mathrm{A}}$ to the energy of the ground state $E_{0}$. The twice excited state with the two quasiparticles having the opposite spins $|2\rangle=\hat{a}_{\uparrow}^{\dagger} \hat{a}_{\downarrow}^{\dagger}|0\rangle$ has the energy

$$
E_{2}=U_{0}+2 \varepsilon_{\mathrm{A}}
$$

In the limit of the infinite gap, one can express the energy of the ground state via the Andreev energy:

$$
U_{0}=\varepsilon_{\mathrm{D}}-\varepsilon_{\mathrm{A}} .
$$

We have omitted the contributions from the resonances that are far below the Fermi level, since they do not influence the formation of the superconductivity and their contribution into $U_{0}$ does not depend upon $\varphi$. Formulas (21)-(24) show that energies $E_{0 / 2}=\varepsilon_{\mathrm{D}} \mp \varepsilon_{\mathrm{A}}$ depend on the phase $\varphi$, while the energy $E_{1}=\varepsilon_{\mathrm{D}}$ does not. The energy levels are presented in Fig. 5 .

\section{The charge of the Andreev quantum dot}

Now we turn to determining the charges of the ground and the excited states and the thermodynamic equilibrium charge. The Andreev state carries a non-trivial charge which can be found as the average of the charge operator in the state $|v\rangle$

$$
\hat{Q}=e \sum_{\sigma} \int_{-L / 2}^{L / 2} \hat{\Psi}_{\sigma}^{\dagger}(x) \hat{\Psi}_{\sigma}(x) d x
$$


where the operator $\hat{\Psi}_{\sigma}$ is defined by the Bogoliubov transformations

$$
\hat{\Psi}_{\sigma}(\mathbf{r})=\sum_{v}\left\{u_{v}(\mathbf{r}) \hat{a}_{v, \sigma}+\operatorname{sign} \sigma v_{v}^{*}(\mathbf{r}) \hat{a}_{v,-\sigma}^{\dagger}\right\}
$$

Correspondingly,

$$
Q_{v}=\langle v|\hat{Q}| v\rangle .
$$

The charge of the state $|v\rangle$ can be also found by differentiating the energy of this state $\varepsilon_{v}$ with respect to the gate potential,

$$
Q_{v}=\frac{\partial E_{v}}{\partial V_{\mathrm{g}}}=e \frac{\partial E_{v}}{\partial \varepsilon_{\mathrm{D}}}
$$

Naturally, both approach yield the same. Hence

$$
Q=Q_{0}=e-Q_{\mathrm{ex}}, \quad Q_{1}=e, \quad Q_{2}=e+Q_{\mathrm{ex}}
$$

where $Q_{\mathrm{ex}}$ is the charge of the single excitation and is equal to the derivative of the Andreev energy $Q_{\mathrm{ex}}=e \partial \varepsilon_{\mathrm{A}} / \partial \varepsilon_{\mathrm{D}}$, see Fig. 6.

For the arbitrary (but small as compared to the adjacent resonances spacing $\delta$ )values of $\Gamma$ and $\varepsilon_{\mathrm{D}}$, the charge is found by the implicit differentiation of Eq. (11), the resulting formula is quite cumbersome and will be analyzed in several particular cases.

The thermodynamic charge is determined by the formula

$$
Q_{\mathrm{eq}}=e+Q_{\mathrm{ex}}\left[1-2 f_{\mathbb{T}}\left(-\varepsilon_{\mathrm{A}}\right)\right]=e+Q_{\mathrm{ex}}\left[f_{\mathbb{T}}\left(\varepsilon_{\mathrm{A}}\right)-f_{\mathbb{T}}\left(-\varepsilon_{\mathrm{A}}\right)\right]
$$

where $f_{\mathbb{T}}(E)$ is the Fermi function with the temperature $\mathbb{T}$,

$$
f_{\mathbb{T}}(E)=\frac{1}{\left(e^{E / k_{\mathrm{B}} \mathbb{T}}+1\right)}
$$

All these charges are localized near the quantum dot, mostly in the interval $[-L / 2 \ldots L / 2]$. The excitation charge $Q_{e x}$ is localized solely in this region and does not change upon expanding integration limits in Eq. (25). The equilibrium charge increases slightly upon increasing the integrating range over the coherence length $\xi$. This can be understood straightforwardly by looking at the times for the quasiparticle to span various parts of the contact. At the quantum point the quasiparticles dwell the lion share of the time $\tau_{\text {dot }} \sim \hbar / \Gamma$, whereas they spend much smaller time $\tau_{\mathrm{Sc}} \sim \xi / v_{\mathrm{F}} \sim \hbar / \Delta$ in the adjacent superconductor. Note that the charge ceases to be localized strictly in a normal region as soon as the Andreev energy becomes of order of the superconducting gap, $\varepsilon_{\mathrm{A}} \approx \Delta$. 

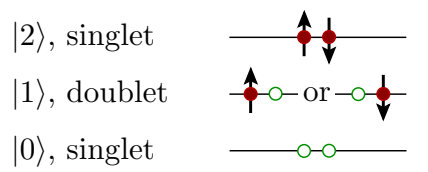

$$
\begin{aligned}
& Q_{2}=Q+2 Q_{\mathrm{ex}}=e+Q_{\mathrm{ex}} \\
& Q_{1}=Q+Q_{\mathrm{ex}}=e \\
& Q_{0}=Q=e-Q_{\mathrm{ex}}
\end{aligned}
$$

Figure 6. Systematics classification of the energy levels in the SINIS contact.

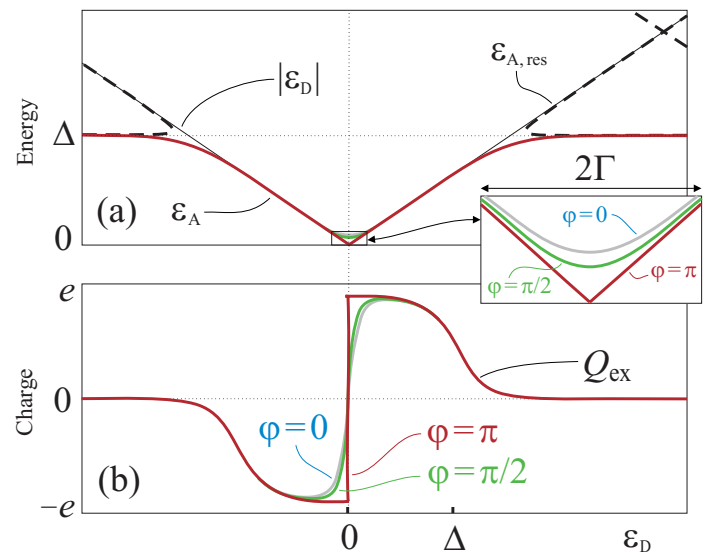

Figure 7. (a) Andreev energy, $\varepsilon_{\mathrm{A}}$ (the red curve), and Andreev resonances, $\varepsilon_{\mathrm{A}, \text { res }}$ (dashed line), in the Andreev dot as functions of the position of the normal resonance $\varepsilon_{\mathrm{D}}$. The half-width of the normal resonance is chosen as $\Gamma=0.1 \Delta$ and symmetric scatterers, $A=0$, are adopted. Shown further are normal resonances, $\left|\varepsilon_{\mathrm{D}}\right|$ (thin black solid line), hole-wise with the negative slope, $\varepsilon_{\mathrm{D}}<0$, electron-wise, with $\varepsilon_{\mathrm{D}}>0$, and in the inset the dependence upon superconducting phase $\varphi$ along the quantum dot. (b) The excitation charge $Q_{\mathrm{ex}}$ is the derivative of the energy with respect to $\varepsilon_{\mathrm{D}}$.

Analogously to the energy, the excitation charge can be analyzed in the different limiting cases:

$$
Q_{\mathrm{ex}} \approx \begin{cases}e \frac{\varepsilon_{\mathrm{D}}}{\sqrt{\varepsilon_{\mathrm{D}}^{2}+\tilde{\Gamma}^{2}}}, & \varepsilon_{\mathrm{D}} \lesssim \Gamma, \\ e \operatorname{sign}\left(\varepsilon_{\mathrm{D}}\right)\left(1-\frac{\Gamma}{\Delta}\right), & \Gamma \lesssim \varepsilon_{\mathrm{D}} \lesssim \Delta, \\ e \operatorname{sign}\left(\varepsilon_{\mathrm{D}}\right) \frac{\Gamma^{2} \Delta}{\varepsilon_{\mathrm{D}}^{3}}, & \Delta \ll \varepsilon_{\mathrm{D}} .\end{cases}
$$

The exact behavior of the $Q(\varphi)$ obtained numerically is shown in Fig. 7(b). One sees that the charge grows linearly with the slope approximately equal to $e / \Gamma \cos (\varphi / 2)$. Note that the dependence becomes the sharp one near $\varphi=\pi$, where the resonance gets across the Fermi level and saturates as $e(1-\Gamma / \Delta)$. As soon as the normal resonance departs from the interval below the gap, $\left|\varepsilon_{\mathrm{D}}\right| \gg \Delta$, the excitation charge decays $\propto 4 \Gamma^{2} \Delta / \varepsilon_{\mathrm{D}}^{3}$. One sees that the fractional charge arises every time as the normal resonance crosses the Fermi level [10]. Note, furthermore, that in addition to the fractional charges corresponding to the ground state and doubly excited (paired) state, there appears an integer charge of a singly excited (unpaired) state $Q_{1}=e$. We are going to focus hereafter on the most interesting case out of 
listed in Eq. (32), corresponding $\varepsilon_{\mathrm{D}} \lesssim \Gamma$, where the charge depends on the $\varphi$. We present it in an explicit form as

$$
Q_{\mathrm{ex}}=e \frac{\varepsilon_{\mathrm{D}}}{\sqrt{\varepsilon_{\mathrm{D}}^{2}+\Gamma^{2}\left(\cos ^{2}(\varphi / 2)+A^{2}\right)}} .
$$

\section{Quantum fluctuations of the charge}

All the above nontrivial charges result from the breaking down the electron-hole symmetry, and are formed as a superposition of electron-wise and hole-wise states with the states corresponding to integer charges. As such, the charges experience quantum fluctuations which are characterized by the mean square deviations

$$
\delta Q_{v}=\sqrt{\left\langle v\left|\hat{Q}^{2}\right| v\right\rangle-\langle v|\hat{Q}| v\rangle^{2}}
$$

An average of $\hat{Q}^{2}$ is given by $\left\langle v\left|\hat{Q}^{2}\right| v\right\rangle=\sum_{v^{\prime}}\left\langle v|\hat{Q}| v^{\prime}\right\rangle\left\langle v^{\prime}|\hat{Q}| v\right\rangle$, where the $v^{\prime}$-summation goes over all the states. We concentrate on fluctuations of the ground, $|0\rangle$, singly excited, $|\uparrow\rangle,|\downarrow\rangle$, and doubly-excited, $|2\rangle$, states. In the experiment the detector measures the charge during some time, $\tau$, thus the measured charge is averaged in time $\bar{Q}=\int_{0}^{\tau}(d t / \tau) Q(t)$; implying that only the matrix elements between the states with the energy difference not exceeding $\hbar / \tau$ are to be taken into account. Assuming that the typical measuring frequencies are all $1 / \tau \ll \Delta / \hbar$, we can restrict the summation over $v^{\prime}$ to summation over the four states of the discrete spectrum $|0\rangle,|\uparrow\rangle,|\downarrow\rangle,|2\rangle$ (in the limit $\Gamma,\left|\varepsilon_{\mathrm{D}}\right| \ll \Delta$ ). In this case these states constitute the complete basis of the Hilbert space, and the only non-diagonal non-zero matrix element is $Q_{02}, Q_{02}=\langle 0|\hat{Q}| 2\rangle=e \sqrt{1-\varepsilon_{\mathrm{D}}^{2} / \varepsilon_{\mathrm{A}}^{2}}$. Then fluctuations of the charge corresponding to the states $|0\rangle$ and $|2\rangle$, we find

$$
\delta Q_{0 / 2}=e \sqrt{1-\varepsilon_{\mathrm{D}}^{2} / \varepsilon_{\mathrm{A}}^{2}}=\sqrt{Q_{0} Q_{2}}
$$

and for $|0\rangle$ we have

$$
\delta Q_{1}=0 .
$$

We see that the charge of the singly-filled state (having an integer charge) does not fluctuate, while the charges of the ground state and doubly excited state (having a fractional charge) fluctuate quite a lot. This illustrates graphically the fact that the cause of the fractional charge in the Andreev dot are fluctuations in the number of particles.

\section{Charge fluctuations due to electron-phonon interaction}

Now we consider the dynamics of the excitation of the Andreev states stemming from thermally induced re-population of the energy levels in the quantum dot. The process of re-population forms a telegraph signal reconfiguring charges in the states $|0\rangle,|1\rangle$, and $|2\rangle$, sketched in Fig. 8. Filling and decay of these states is accompanied by emission 


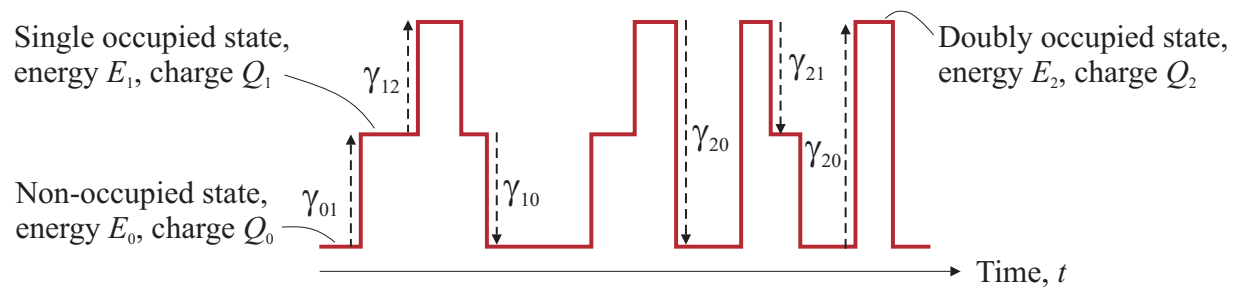

Figure 8. The structure of the telegraph process due to excitations of Bogoliubov quasiparticles by phonons. Transitions changing the system state over the even number of quasiparticles, $0 \leftrightarrow 2$ are fast, whereas the changes over the odd number of quasiparticles, $0 \leftrightarrow 1$ and $1 \leftrightarrow 2$ ), are slow.

and absorption of phonons [36]. The contribution into re-population comes from two processes, transitions between the ground and singly excited states with frequencies $\gamma_{01}$ and $\gamma_{10}=\gamma_{01} \exp \left(\varepsilon_{\mathrm{A}} / \mathbb{T}\right)$, respectively, and transitions between the ground- and twice excited states with frequencies $\gamma_{02}$ and $\gamma_{20}=\gamma_{02} \exp \left(2 \varepsilon_{\mathrm{A}} / \mathbb{T}\right)$, respectively. Note that transitions $0 \leftrightarrow 1$ and $1 \leftrightarrow 2$ are equivalent and have same frequencies, $\gamma_{01}=\gamma_{12}$ and $\gamma_{10}=\gamma_{21}$. Transitions $0 \leftrightarrow 1$ occur via emission of the extra quasiparticle with the energy $E$ belonging to continuous spectrum, $E>\Delta$.

One can find the frequencies $\gamma_{01}$ and $\gamma_{02}$ making use of Fermi golden rule with the Hamiltonian describing electron-phonon interaction

$$
\hat{\mathcal{H}}_{\mathrm{el}-\mathrm{ph}}=g \int d x \hat{n}_{e}\left(\partial_{x} u\right)
$$

where $u$ is the displacement, $n_{e}$ is the electron density, and $g$ is the electron-phonon coupling constant typically of order of $1 \mathrm{eV}$. We will consider $1 \mathrm{D}$ electron and phonon modes. The direct calculation of frequency $\gamma_{02}$ due to electron-phonon interaction yields

$$
\gamma_{02} \sim\left(g^{2} / \hbar m v_{\mathrm{F}}^{2}\right)\left(a / k_{\mathrm{ph}} L^{2}\right) N_{\mathbb{T}}\left(2 \varepsilon_{\mathrm{A}}\right),
$$

where $N_{\mathbb{T}}(\epsilon)$ is the Bose function of the phonon states corresponding the temperature $\mathbb{T}, a$ is the lattice constant, $k_{\mathrm{ph}}=2 \varepsilon_{\mathrm{A}} / \mathrm{s}$ is the phono wave vector corresponding to the transition energy $2 \varepsilon_{\mathrm{A}}$, and $s$ is the sound velocity. Equation (38) is valid for 1D phonons provided their wavelength is less than the contact length, and, accordingly, $k_{\mathrm{ph}} \gg 1 / L$. In the opposite case, $k_{\text {ph }} \ll 1 / L$ one has 0 -dimensional situation giving rise to

$$
\gamma_{02} \sim\left(g^{2} / \hbar m v_{\mathrm{F}}^{2}\right) a k_{\mathrm{ph}} N_{\mathbb{T}}\left(2 \varepsilon_{\mathrm{A}}\right)
$$

Numerical estimate with $g \sim 1 \mathrm{eV}, m v_{\mathrm{F}}^{2} \sim 1 \mathrm{eV}$, and $L \approx 500 \mathrm{~nm}$ [27] yields $\gamma_{02} \sim$ $N_{\mathbb{T}}\left(2 \varepsilon_{\mathrm{A}}\right) 10^{12} \mathrm{sec}^{-1}$ for $k_{\mathrm{ph}} \sim 1 / L$.

Calculation of the frequency $\gamma_{01}$ requires additional summation over the states of the continuous spectrum with $E>\Delta$. This summation is dominated by the states with energies $E \sim \Delta$. Estimating the frequency of simultaneous filling the Andreev level and the state 
belonging in the continuous spectrum which is accompanied by absorption of the phonon and decay of the Cooper pair, we find

$$
\gamma_{01} \sim\left(g^{2} T_{1} / \hbar m v_{\mathrm{F}}^{2}\right)\left(a s / L v_{\mathrm{F}}\right) \sqrt{k_{\mathrm{B}} \mathrm{T} / \Delta} e^{-\Delta / k_{\mathrm{B}} \mathrm{T}}\left[1+e^{-\varepsilon_{\mathrm{A}} / k_{\mathrm{B}} \mathrm{T}}\right]
$$

Here $k_{\mathrm{ph}} L \sim \Delta L / \hbar s \gg 1$ and $T_{1}$ is the transparency of a single barrier. We consider here a symmetric SINIS contact, $T_{1}=T_{2}$. Using the typical values $k_{\mathrm{B}} \mathbb{T} \approx 0.1 \Delta \approx 1 \mathrm{~K}$ and $v_{\mathrm{F}} / s \sim 10^{3}$, we estimate $\gamma_{01} \sim T_{1} \exp \left(-\Delta / k_{\mathrm{B}} \mathrm{T}\right) 10^{10} \mathrm{sec}^{-1}$.

Three-dimensionality of the phonon modes results in the additional factor

$$
\left(\varepsilon_{\mathrm{A}} / \hbar \omega_{\mathrm{D}}\right)^{3}(L / a) \sim 10^{-6}
$$

reducing frequency (38). The frequency (40) is reduced by the factor

$$
\left(\Delta / \hbar \omega_{\mathrm{D}}\right)^{3}(L / a) \sim 10^{-3}
$$

where $\omega_{\mathrm{D}}$ is Debye frequency and we assume $\varepsilon_{\mathrm{A}} / \hbar \omega_{\mathrm{D}} \sim 10^{-3}$.

One sees that the process $0 \leftrightarrow 2$ is more frequent than the process $0 \leftrightarrow 1$, since the latter includes the exponential factor $\exp (-\Delta / \mathbb{T})$, which allows reduction of the frequency $\gamma_{01}$, by lowering the temperature, see Fig. 8.

\section{Fluctuations of the gate potential}

An additional contribution to re-populating levels comes from the fluctuations of the gate voltage $V_{\mathrm{g}}$. These fluctuations are defined by the interaction Hamiltonian $\hat{\mathcal{H}}_{\mathrm{g}}=\int d x e \hat{n}_{e} V_{g}$. Making use of the technique analogous to that of the previous sections, one finds the respective transition frequencies. Transitions Transitions $0 \leftrightarrow 2$ occur with frequency

$$
\tilde{\gamma}_{02} \sim\left(e^{2} / \hbar C\right) N_{\mathbb{T}}\left(2 \varepsilon_{\mathrm{A}}\right)
$$

where $C$ is the contact capacitance to the gate. Taking $C \sim L=500 \mathrm{~nm}$, one finds the frequency of the gate fluctuations $\tilde{\gamma}_{02} \sim N_{\mathbb{T}}\left(2 \varepsilon_{\mathrm{A}}\right) 10^{11} \mathrm{sec}^{-1}$, which compares to the similar contribution from phonons $\gamma_{02}$. Transitions due to gate potential fluctuations accounting for the continuous spectrum yield frequencies of the transitions in which the quasiparticle number parity changes

$$
\tilde{\gamma}_{01} \sim \hbar^{-1}\left(e^{2} / C\right) \sqrt{k_{\mathrm{B}} \mathbb{T} / \Delta} e^{-\Delta / k_{\mathrm{B}} \mathbb{T}}\left[1+e^{-\varepsilon_{\mathrm{A}} / k_{\mathrm{B}} \mathbb{T}}\right]
$$




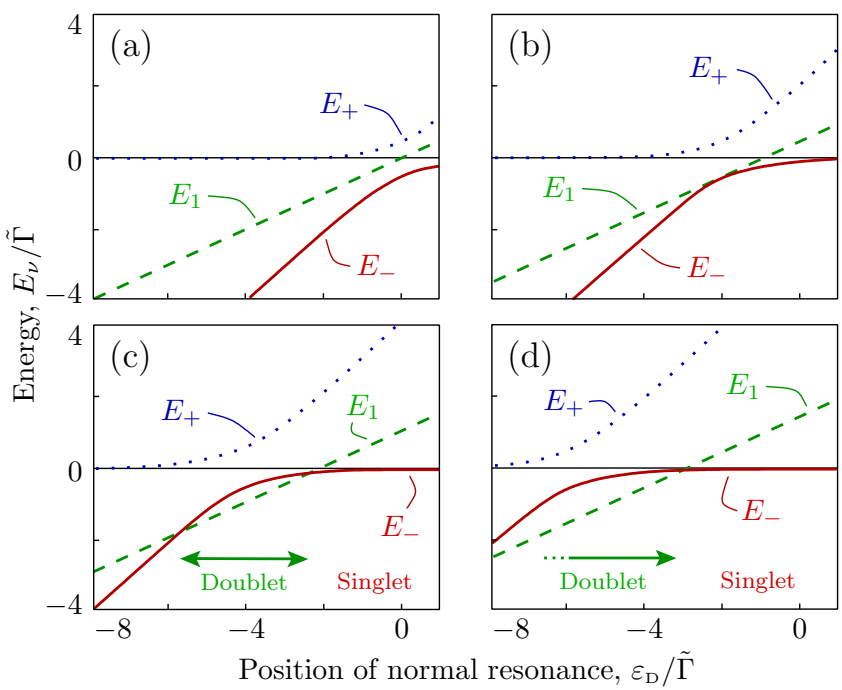

Figure 9. Energies $E_{-}$(the red solid line), $E_{1}$ (the green dashed line), and $E_{+}$(blue dotted line) as functions of the position of the normal resonance $\varepsilon_{\mathrm{D}}$, all energies being presented in the units $\tilde{\Gamma}$, see Eq. (15). The Coulomb energy $E_{C}=0$ for $(\mathrm{a}), E_{\mathrm{C}}=\tilde{\Gamma}$ for $(\mathrm{b}), E_{\mathrm{C}}=2 \tilde{\Gamma}$ for $(\mathrm{c})$, and $E_{\mathrm{C}}=3 \tilde{\Gamma}$ for $(\mathrm{d})$. At $E_{\mathrm{C}} \geqslant \tilde{\Gamma}$, the region exists where where the ground state is the doublet (shaded in gray). The width of the doublet domain is $2 \sqrt{E_{C}^{2}-\tilde{\Gamma}^{2}}$, the boundaries are blurred by the finite temperature $\mathbb{T}$.

Plugging in the typical values of parameters $k_{\mathrm{B}} \mathbb{T} \approx 0.1 \Delta \approx 1 \mathrm{~K}$ and $C \sim L$, we get $\tilde{\gamma}_{01} \sim$ $\exp \left(-\Delta / k_{\mathrm{B}} \mathrm{T}\right) 10^{11} \mathrm{sec}^{-1}$. Similarly to the case of phonons, we observe that the process $0 \leftrightarrow 2$ always happens more often than $0 \leftrightarrow 1$ due to $\exp \left(-\Delta / k_{\mathrm{B}} \mathbb{T}\right)$ factor.

\section{Coulomb interaction}

To take into account the Coulomb interaction we have to take into account the charge screening at the quantum dot and mixing of the charge states. The screening due to an additional charge tunneling into the Andreev quantum dot is determined by the density of states. The corresponding energy scale is set by the gap between the adjacent resonances $\delta$ since each of them carries the charge $2 e$. As a result the screening at the quantum dot is not important provided the Coulomb energy is smaller than the separation between the resonances, $E_{\mathrm{C}} \ll \delta$. The scale $E_{\mathrm{C}} \approx e^{2} / 2 C$ can be estimated taking the capacitance of the quantum $\operatorname{dot} C=\epsilon L$, where $\epsilon$ is the dielectric permeability of the medium. On the other hand, the separation between the adjacent resonances $\delta=h v_{\mathrm{F}} / 2 L$, is inversely proportional to the normal part length $L$ as well as $E_{\mathrm{C}}$. The key parameter is the dimensionless ratio $\delta / E_{\mathrm{C}}=h v_{\mathrm{F}} \epsilon / e^{2}$. Taking typical $\epsilon \sim 10$ and $v_{\mathrm{F}} \sim 10^{6} \mathrm{~m} / \mathrm{sec}$, we obtain $\delta / E_{\mathrm{C}} \approx 30$, so we can order energies as $E_{\mathrm{C}} \lesssim \Delta \lesssim \delta$. The paper [27] addressed the Andreev quantum dot formed by the nanotube of the length $L \approx 500 \mathrm{~nm}$, corresponding the Coulomb energy $E_{\mathrm{C}} \sim 1 \mathrm{~K}$. Thus the above chain of inequalities is experimentally feasible.

To investigate mixing of the charge states in the limit $E_{C}, \Gamma,\left|\varepsilon_{\mathrm{D}}\right| \ll \Delta$, we again restrict ourselves to contribution of the four states: $|0\rangle,|\uparrow\rangle,|\downarrow\rangle$, and $|2\rangle$. The interaction is defined 
by the operator

$$
\hat{V}=E_{\mathrm{C}} \frac{\hat{Q}^{2}}{e^{2}} .
$$

In the basis of these for states, the diagonalization can be performed exactly. The non-zero matrix elements of $\hat{V}$ are

$$
V_{00}=E_{\mathrm{C}} \frac{Q_{0}^{2}+Q_{02}^{2}}{e^{2}}, \quad V_{11}=E_{\mathrm{C}}, \quad V_{22}=E_{\mathrm{C}} \frac{Q_{2}^{2}+Q_{02}^{2}}{e^{2}}, \quad V_{02}=2 E_{\mathrm{C}} \frac{Q_{02}}{e} .
$$

New energy levels are determined from the consistency condition for the following system of equations:

$$
\left[\begin{array}{cccc}
\tilde{\varepsilon}_{0}-E & & & V_{02} \\
& \tilde{\varepsilon}_{1 \uparrow}-E & & \\
& & \tilde{\varepsilon}_{1 \downarrow}-E & \\
V_{20} & & & \tilde{\varepsilon}_{2}-E
\end{array}\right]\left[\begin{array}{l}
D_{0} \\
D_{\uparrow} \\
D_{\downarrow} \\
D_{2}
\end{array}\right]=0,
$$

where we introduced notations $\tilde{\varepsilon}_{v}=\varepsilon_{v}+V_{v v}$ with $v=0, \uparrow, \downarrow, 2$. The energy of the state with a single Bogoliubov quasiparticle $|1\rangle$ sifts over the constant equal the the Coulomb energy

$$
E_{1}=\varepsilon_{\mathrm{D}}+E_{\mathrm{C}}
$$

and the state itself $|1\rangle$ does not mix with the other states and retains its degeneracy with respect to spin. This state is called Kramers doublet. The ground state $|0\rangle$ and twice degenerate and twice excited $|2\rangle$ states do mix due to Coulomb interaction and generate two new singlet states $|-\rangle$ and $|+\rangle$. The new states are expressed through the coefficients $D_{0}, D_{\uparrow}, D_{\downarrow}$, and $D_{2}$ in Eq. (47) as follows: $| \pm\rangle=D_{0}^{ \pm}|0\rangle+D_{2}^{ \pm}|2\rangle$. The energies of new states are

$$
E_{ \pm}=\varepsilon_{\mathrm{D}}+2 E_{\mathrm{C}} \pm \sqrt{\left(\varepsilon_{\mathrm{D}}+2 E_{\mathrm{C}}\right)^{2}+\tilde{\Gamma}^{2}}
$$

The energies of the doublet and singlet states depend on the position of the normal resonance $\varepsilon_{\mathrm{D}}$ and the difference of the superconducting phases $\varphi$ differently, and at some values of $\varepsilon_{\mathrm{D}}$ and $\varphi$ the situation can occur where $E_{-}>E_{1}$. Therefore the ground state can be created by either singlet, $|-\rangle$, or doublet, $|1\rangle$, states and the state $|+\rangle$ always remains doubly excited, see Fig. 9. If $E_{\mathrm{C}}<\tilde{\Gamma}$, the ground state is always formed by the singlet $|-\rangle$. Otherwise, if $E_{\mathrm{C}} \geqslant \tilde{\Gamma}$, the ground state is formed by the doublet $|1\rangle$ in the region

$$
-2 E_{\mathrm{C}}-\sqrt{E_{\mathrm{C}}^{2}-\tilde{\Gamma}^{2}}<\varepsilon_{\mathrm{D}}<-2 E_{\mathrm{C}}+\sqrt{E_{\mathrm{C}}^{2}-\tilde{\Gamma}^{2}}
$$




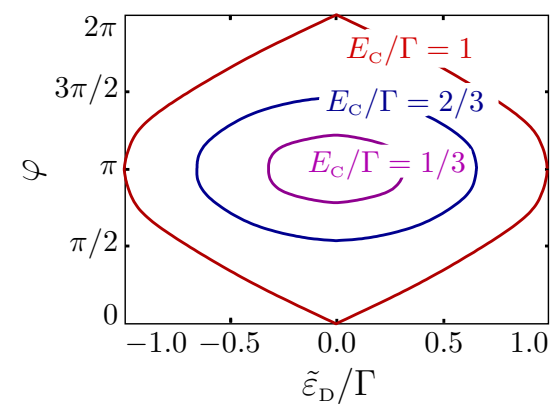

Figure 10. The doublet region in the coordinates $\left(\varphi, \tilde{\varepsilon}_{D}\right)$, where $\tilde{\varepsilon}_{D}=\varepsilon_{D}+2 E_{C}$ is position of the normal resonance shifted by the Coulomb interaction. The size of the doublet region grows upon the increase in the Coulomb energy $E_{\mathrm{C}}$. $E_{\mathrm{C}} / \Gamma=1 / 3$ (magenta), 2/3 (blue), and 1 (red).

or, which is the same,

$$
\left(\varepsilon_{\mathrm{D}}+2 E_{\mathrm{C}}\right)^{2}+\Gamma^{2} \cos ^{2} \frac{\varphi}{2}+\Gamma^{2} A^{2}<E_{\mathrm{C}}^{2}
$$

and remains a singlet $|-\rangle$ at all other values $\varepsilon_{\mathrm{D}}$ [37]. At the boundary of the region (50) occurs the transition from the singlet into the doublet state, the charge of the Andreev quantum dot and the current change abruptly as a jump.The boundary of the doublet region in the variables $\left(\varphi, \varepsilon_{\mathrm{D}}\right)$ is shown in Fig. 10.

The charges of the new states can be calculated as derivatives of the corresponding energies with respect to the gate voltage $Q_{v}=\partial E_{v} / \partial V_{\mathrm{g}}$ :

$$
Q_{ \pm}=e\left(1 \pm \frac{\varepsilon_{\mathrm{D}}+2 E_{\mathrm{C}}}{\sqrt{\left(\varepsilon_{\mathrm{D}}+2 E_{\mathrm{C}}\right)^{2}+\tilde{\Gamma}^{2}}}\right), \quad Q_{1}=e .
$$

Everywhere except for the doublet region the charge of the ground state is equal to $Q_{-}$, while in the doublet region the charge is $Q_{1}=e$. One can observe from Fig. 11(a) and 11(b), that if the Coulomb energy exceeds the critical value $E_{\mathrm{C}}>E_{\mathrm{C}}^{*}=\Gamma A$, the ground state reconstructs itself into a doublet one. The charge during this process jumps over $Q_{-}-Q_{1}$, at finite temperature the transition is being smoothed, see Fig. 11(c) and 11(d). The thermodynamic equilibrium charge at finite temperature, $\mathbb{T}$, is

$$
Q_{\mathrm{eq}}=\frac{Q_{-} e^{-E_{-} / k_{\mathrm{B}} \mathbb{T}}+2 Q_{1} e^{-E_{1} / k_{\mathrm{B}} \mathbb{T}}+Q_{+} e^{-E_{+} / k_{\mathrm{B}} \mathbb{T}}}{e^{-E_{-} / k_{\mathrm{B}} \mathbb{T}}+2 e^{-E_{1} / k_{\mathrm{B}} \mathbb{T}}+e^{-E_{+} / k_{\mathrm{B}} \mathrm{T}}} .
$$

The charge of the Andreev quantum dot exhibits nontrivial dependencies on the difference of the superconducting phases between the banks. The discussed effects hold high potential for applications in various nanodevices. 

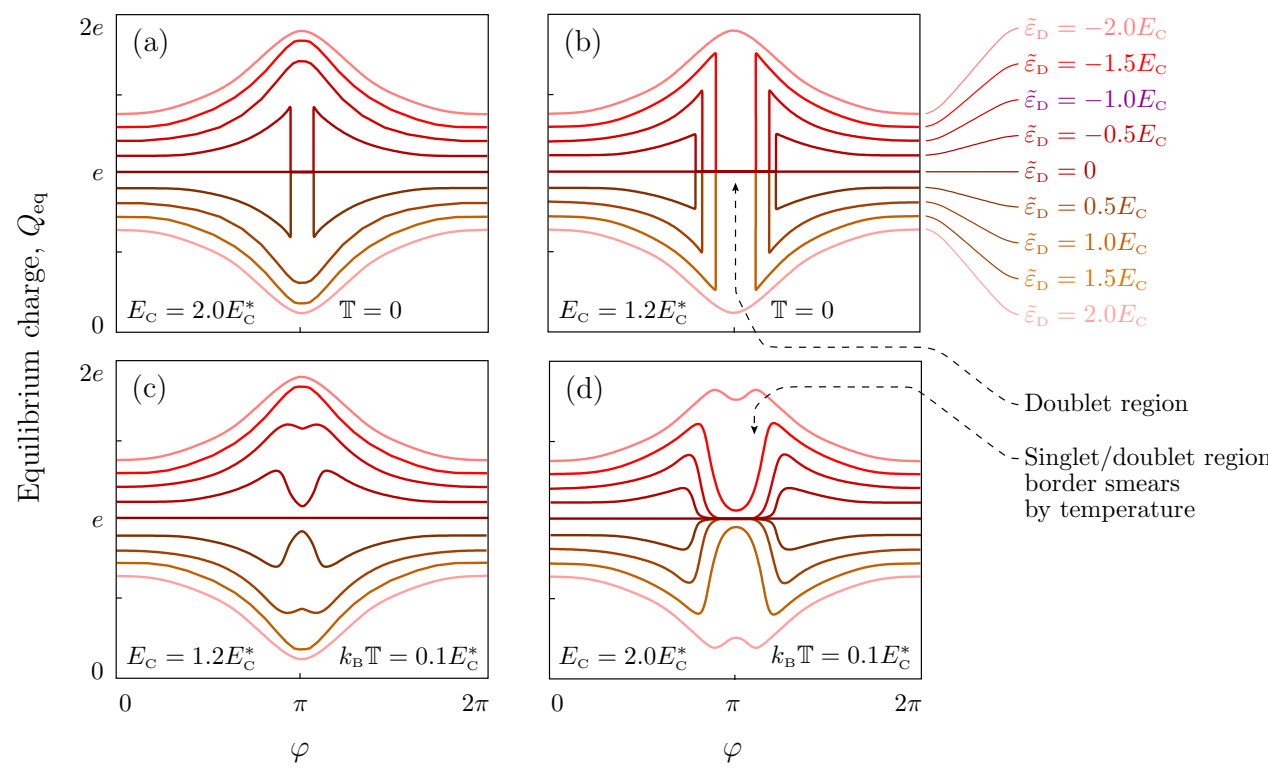

Figure 11. The equilibrium charge $Q_{\mathrm{eq}}$ as function of a difference of superconducting phases $\varphi$. The plots (a) and (b) correspond to zero temperature, i.e. $Q_{\text {eq }}$ is equal to the charge of the ground state. Plots (c) and (d) correspond to temperature $k_{\mathrm{B}} \mathbb{T}=0.1 E_{\mathrm{C}}^{*}$, where $E_{\mathrm{C}}^{*}=\Gamma A$. The Coulomb energy is $E_{\mathrm{C}}=1.2 E_{\mathrm{C}}^{*}$ in panels (a) and (c), and $E_{\mathrm{C}}=2.0 E_{\mathrm{C}}^{*}$ in panels (b) and (d). The asymmetry of the quantum $\operatorname{dot} A=0.2$. The singularities in the centers of the plots correspond to the doublet region (50). In panels (c) and (d) the boundary of the doublet region is blurred by temperature $\mathbb{T}$.

\section{Scaling of the charge}

We were assuming above that there exists a single conducting channel with the spin. Let us ask how would the charge scale if we had several conducting channels. For example, even in the case of the single-wall carbon nanotube, there is an orbital degeneracy and, in general, there are two channels with the spin. In the case of the multi-wall nanotube (or the normal metal) there may be more than one channel. In this case the Coulomb interaction between the different channels gives rise to the non-trivial charge states [30]. Here we restrict ourselves to a more simple case of multiple channels, but neglecting Coulomb effects. We investigate the SNS contacts endowed with the quadratic and linear dispersions in the normal part, corresponding to the contact superconductor-graphene-superconductor (SGS) [38].

We have demonstrated above that the phase-dependent part of the SINIS charge with the strong normal resonance change the sign upon traversing this resonance across the Fermi level. It is clear that in the absence of the Coulomb effects the contributions from the different channels are additive. This implies that if the part of the resonances are above Fermi level and other resonances are below it, then their contributions to the phase-sensitive part of the charge do compensate one another. As we have already mentioned, the situation where a group of the resonances appears either only above (or only below) the Fermi level is possible due to inhomogeneous distribution of the energy levels due to lateral quantization in the normal part [39]. 
Let us consider a short SNS contact (so that $L \ll \xi$ )with the ideal NS boundaries without normal resonances. In the case of the absence of resonances the maximal sensitivity of a charge per one channel is much less than that in the presence of the resonances. At resonances the contribution into the charge from a single channel is of order of electron charge $e[10,22]$, whereas in the case of the ideal boundaries, it is of order $\left(\Delta / E_{\mathrm{F}}\right) e \sim$ $10^{-4} e$ [11]. However, the contribution from these channels comes with the like sign, and there may be many of such channels.

The Andreev levels in the short SNS contact are given by

$$
\varepsilon_{\mathrm{A}, v}(\varphi, \mu)=\Delta \sqrt{1-T_{v} \sin ^{2}(\varphi / 2)},
$$

where $T_{v}$ is the transparency of the normal region. This formula assumes that $T_{v}$ remains constant and does not depend on energy in the interval $[\mu-\Delta \ldots \mu+\Delta]$, which is equivalent to inequality $\Gamma_{v} \gg \Delta$, where $\Gamma_{v}$ is the half width of the resonance having transparency $T_{v}$. The width of the contact we denote by $W$. Now, the phase-sensitive part of the charge $Q(\varphi, \mu)$ hybridized in the contact can be calculated by summation over all the channels

$$
Q(\varphi, \mu)=-2 e \sum_{\nu=0}^{\infty} \frac{\partial}{\partial \mu} \varepsilon_{\mathrm{A}, \nu}(\varphi, \mu) .
$$

The factor 2 results from the degeneracy with respect to spin. Note that in Eq. (55) we perform summation over both, propagating modes (when the energy of the lateral quantization is less than the Fermi energy and the solutions of the quasi-1D wave equation has a form of non-decaying waves), and over the dissipative modes (with the energies of the lateral quantization exceeding the Fermi energy and accordingly, decaying solutions of the wave equations). In the case of the linear dispersion only the propagation modes appear relevant.

\subsection{Model rectangular potential}

To begin with, let us present the distribution of the channels over the energies with the aid of the set of rectangular potentials

$$
U_{v}(x)=\left[E_{\mathrm{F}}-\mu_{v}\right] \Theta(L / 2-|x|)
$$

(see inset in Fig. 12(a)) with the corresponding transparencies.

$$
T_{v}(\mu)=\frac{4 E_{\mathrm{F}} \mu_{v}}{4 E_{\mathrm{F}} \mu_{v}+\left(E_{\mathrm{F}}-\mu_{v}\right)^{2} \sin ^{2}\left[\left(2 m \mu_{v} / \hbar^{2}\right)^{1 / 2} L\right]},
$$

where $E_{\mathrm{F}}$ is the Fermi energy in the bulk superconductor, and $\mu_{v}=\mu-\pi^{2} \hbar^{2} v^{2} / 2 m W^{2}$ is the effective Fermi level in the normal part for $v$-th mode, which one can tune changing 
the gate potential $V_{\mathrm{g}}: \mu=E_{\mathrm{F}}-e V_{\mathrm{g}}$. Positive $\mu_{v}$ correspond to the propagating modes, while the negative $\mu_{v}$ denote the decaying modes. The number of the propagating modes $N=\left[\sqrt{2 m \mu_{v}} W / \pi \hbar\right]$, where square brackets [...] denote the integer part of the real part. Replacing summation over the modes by integration in Eq. (55), introducing the dimensionless parameters $\lambda=2 m \mu L^{2} / \hbar^{2}, \Lambda=2 m E_{\mathrm{F}} L^{2} / \hbar^{2}$ and $x=\sqrt{\left|1-\mu_{\nu} / \mu\right|}=$ $\sqrt{\pi^{2} \hbar^{2} / 2 m W^{2}|\mu|} v$, we arrive at the following transparency

$$
T(\lambda)=\frac{4 \Lambda \tilde{\lambda}}{4 \Lambda \tilde{\lambda}+[\Lambda-\tilde{\lambda}]^{2} \sin ^{2} \tilde{\lambda}^{1 / 2}}
$$

and charge

$$
Q(\varphi, \mu)=-2 e \frac{2 m L W \Delta}{2 \pi \hbar^{2}} \int_{0}^{\infty} \frac{d x}{\sqrt{|\lambda|}}\left\{\sqrt{1-T \sin ^{2}(\varphi / 2)}-\frac{\sin ^{2}(\varphi / 2)}{\sqrt{1-T \sin ^{2}(\varphi / 2)}} \lambda \frac{\partial T}{\partial \lambda}\right\}
$$

where $\tilde{\lambda}=\lambda\left(1-x^{2} \operatorname{sign} \lambda\right)$.

The charge determined by the last formula is shown in the Fig. 12(a) for $\Lambda=10^{2}$ and 12(b) for $\Lambda=10^{4}$. Non-monotonic dependence upon the position of the gate stems from the resonances in the transparency (57). In the real experiment the potential is not sharp and its structure is significantly blurred.

\subsection{Parabolic potential}

The model that describes qualitatively the electrostatics of the gate results in the parabolic potential. This potential is universal in a sense that its transparency near the top depends only on its "curvature" $\partial_{x}^{2} U_{n}(x)$. In a short contact where one can neglect by the difference between the mean free times of electrons and holes over the normal part, this universality maintains for SNS contact with the parabolic potentials inside.
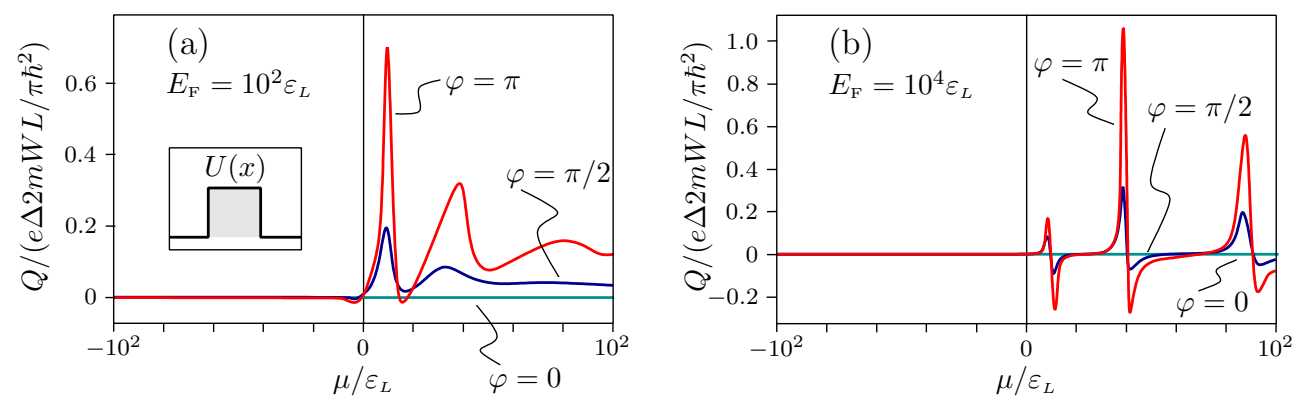

Figure 12. The phase-sensitive part of the charge $Q(\varphi, \mu)$ of the SNS contact as a function of the effective Fermi energy $\mu$ in the normal region at different superconducting phases $\varphi=0, \pi / 2, \pi$. (a) The Fermi energy in a superconductor $E_{\mathrm{F}}=10^{2} \varepsilon_{L}$. (b) The Fermi energy $E_{\mathrm{F}}=10^{4} \varepsilon_{L}$. 

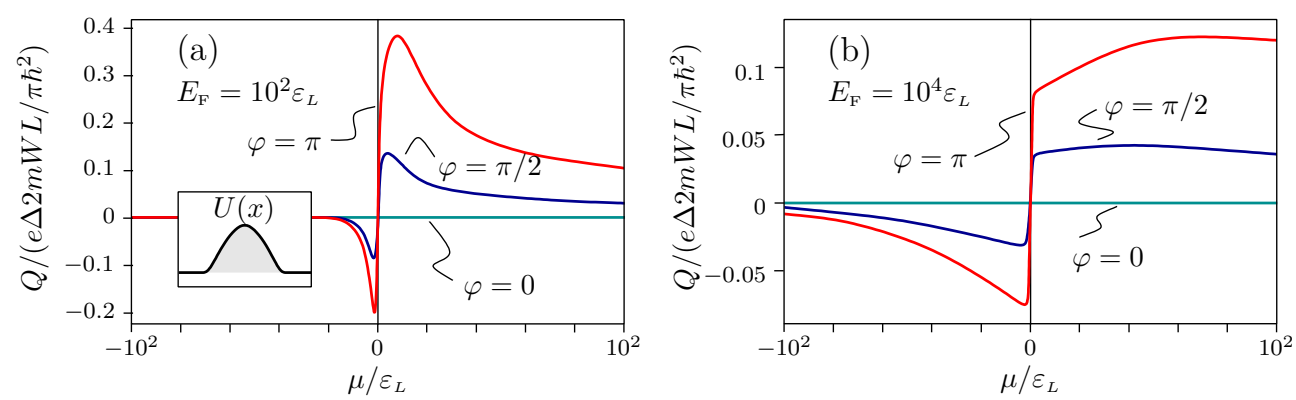

Figure 13. The same as in Fig. 12, but for the effective Fermi energy of a parabolic shape.

Let us consider the parabolic barrier corresponding the the $v$-th mode:

$$
U_{v}(x)=\left(E_{\mathrm{F}}-\mu_{v}-\frac{m \Omega_{v}^{2} x^{2}}{2}\right) \Theta(L / 2-|x|)
$$

with the curvature at the maximum $\Omega_{v}=(4 / \hbar) \sqrt{\varepsilon_{L}\left(E_{\mathrm{F}}-\mu_{v}\right)}$, see inset in Fig. 13(a). As before, $\varepsilon_{L}=\hbar^{2} / 2 m L^{2}$. The potential is chosen to correspond the condition $U_{v}( \pm L / 2 \mp 0)=0$ at NS boundaries. The transparency near the maximum is given by the Kemble formula

$$
T_{v}(\mu)=\frac{1}{1+\exp \left(-2 \pi \mu_{v} / \hbar \Omega_{v}\right)}
$$

Making use the same parametrization as for a rectangular barrier, we find

$$
T(\lambda)=\frac{1}{1+\exp (-2 \pi \tilde{\lambda} / \sqrt{\Lambda-\tilde{\lambda}})}
$$

(the formula for the charge looks alike). The phase-sensitive part of the charge is shown in Figs. 13(a) and 13(b). This part of the charge changes sign at $\mu=0$ and mostly is monotonic as predicted. The "amplitude" of the phase-dependent part of the charge is expressed through the coherence length $\xi=\hbar v_{\mathrm{F}} / \Delta$, giving rise to

$$
Q \sim \frac{2|e|}{\pi} \frac{W}{\xi} \sqrt{\frac{E_{\mathrm{F}}}{\varepsilon_{L}}}=\frac{2|e|}{\pi} \frac{W}{\xi}
$$

We see that the part of the charge we are interested in is proportional to the width of the normal part of the contact $W$, and that the magnitude of the effect is controlled mostly by the ratio $W / \xi$. 


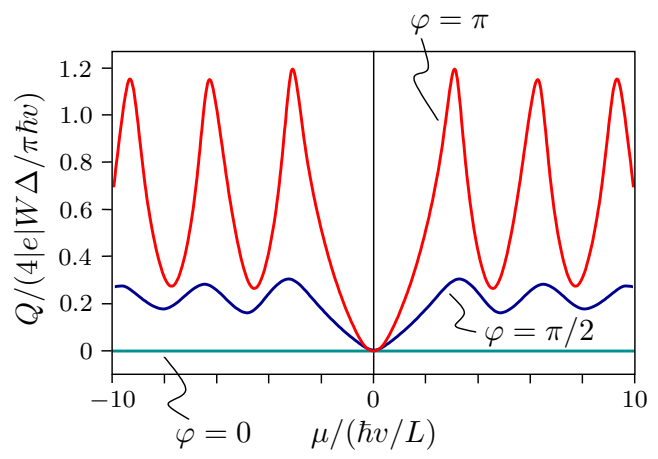

Figure 14. The charge of the SGS contact.

\subsection{Graphene stripe with linear dispersion}

The contacts of the SGS type are characterized by the linear dispersion. The critical currents across these contacts was studied theoretically [40,41] and experimentally [38]. In the case of graphene we will be solving Dirac-Bogoliubov-de Gennes equations [42], rather than the usual Bogoliubov-de Gennes ones. For the wide, $W \gg L$, and short, $L \ll \xi$, rectangular sheets of graphene (here as well as in the previous subsection, $L$ and $W$ are the length and the width of the normal region, and $\xi=\hbar v / \Delta$ is the coherence length in superconductors.), the Andreev energy is given by Eq. (54) with the new transparency [40]:

$$
T_{v}(\mu)=\frac{\mu_{v}^{2}}{\mu_{v}^{2}+\left(\mu^{2}-\mu_{v}^{2}\right) \sin ^{2}\left[\left(\mu_{v} / \hbar v\right) L\right]},
$$

where $\mu_{v}^{2}=\mu^{2}-[\hbar v(v+1 / 2) \pi / W]^{2}$. Here the real $\mu_{v}$ correspond to the propagating modes, while the imaginary ones, describe the decaying modes. The charge of the dot is given by the expression

$$
\tilde{Q}(\varphi, \mu)=-4 e \frac{\partial}{\partial \mu} \sum_{\nu=0}^{\infty} \varepsilon_{\mathrm{A}, v}(\varphi, \mu) .
$$

The prefactor 4 arises in the last three formulas due to a double degeneracy with respect to spins and volleys in the graphene. We are still interested only by the phase-sensitive part of the charge. Introducing new variables, $x=(v+1 / 2) \pi \hbar v /|\mu| W$, and $\lambda=\mu L / \hbar v$, we find

$$
T(\lambda)=\frac{\tilde{\lambda}^{2}}{\tilde{\lambda}^{2}+\left(\lambda^{2}-\tilde{\lambda}^{2}\right) \sin ^{2} \tilde{\lambda}^{\prime}}
$$

where, as before, $\tilde{\lambda}=\lambda\left(1-x^{2} \operatorname{sign} \lambda\right)$. For a wide contact we can replace summation by the integration to obtain: 


$$
\tilde{Q}(\varphi, \mu)=-4 e \frac{W \Delta}{\pi \hbar v} \int_{0}^{\infty} d x \frac{\partial}{\partial \lambda}\left[\lambda \sqrt{1-T \sin ^{2} \frac{\varphi}{2}}\right]
$$

The integral in Eq. (67) diverges, but, since we are interested only by the phase-sensitive part of the charge, we can extract unity from the integrand to get:

$$
Q(\varphi, \mu)=-4 e \frac{W \Delta}{\pi \hbar v} \int_{0}^{\infty} d x\left[\sqrt{1-T \sin ^{2} \frac{\varphi}{2}}-1+\frac{\sin ^{2}(\varphi / 2)}{2 \sqrt{1-T \sin ^{2}(\varphi / 2)}} \lambda \frac{\partial T}{\partial \lambda}\right]
$$

This charge is shown in Fig. 14. One sees that the integral in Eq. (68) varies from zero to some number of order unity.

Let us compare the factors in front of the integrals in expressions for SNS and SGS contacts. Both are proportional to the width of the contact $W$, but in case SNS contact the factor does not depend on $\mu$. Because of the rectangular graphene sheet we observe oscillations. The phase-sensitive part of the charge is of order $Q \sim 4|e| W \Delta / \pi \hbar v$. Expressing it through the superconducting coherence length $\xi$, we get

$$
Q \sim \frac{4|e|}{\pi} \frac{W}{\xi}
$$

We see that the effect is again proportional to the width $W$ and is controlled by the ratio $W / \xi$.

\section{Measuring of the fractional charge}

The charge can be measured by the capacitance technique tying the charge detector, such as single-electron transistor [21, 43] to the Andreev quantum dot. This approach suffers some difficulties due to, for example, fluctuations of the charges of substrate and the gate discussed in the Sec. 7. Another important problem is the such a measurement "feels" not the total charge $Q$ of the Andreev quantum dot, bit only its fraction $\alpha_{\mathrm{C}} \alpha_{\mathrm{S}} Q$.

The geometry factor $\alpha_{\mathrm{C}}$ is determined by the detail of the measuring gate. Let the mutual capacitance of the quantum dot and the measuring contact be $C_{\mathrm{m}}$, and the mutual capacitance of the Andreev quantum dot and the rest contacts as $C_{\mathrm{o}}$. Then $\alpha_{\mathrm{C}}=C_{\mathrm{m}} /\left(C_{\mathrm{m}}+C_{\mathrm{o}}\right)$. The factor $\alpha_{\mathrm{S}}=\alpha_{\mathrm{S}}(Q)$ is determined by the dynamic feedback from the charge detector. Here we will assume $\alpha_{\mathrm{C}}=\alpha_{\mathrm{S}}=1$, but bear in mind that the measurement would give us only a part of the charge. One of the most essential aspects of this work is the fact that the charge depends on the phase difference at the banks. Thus the important characteristic of the measurements is the differential sensitivity of the charge with respect to the phase.

$$
\mathcal{S}=\frac{2 e}{\hbar} \frac{\partial Q}{\partial \varphi}
$$


Fluctuations of the charge due to re-population of the levels, generally speaking, interfere in the observation of the charge of the ground and the excited states, however, in certain regimes they can be used for the detecting the charge. The signal resolution of the up-to-date single electron transistors is about $\sim 10^{-5}|e| / \mathrm{Hz}^{1 / 2}$ at frequencies $f<10^{9} \mathrm{~Hz}$ [43]; therefore indeed the telegraph signal due to thermodynamic re-population of the Andreev state can be resolved, accordingly, the fractional charge due to thermodynamic re-population can be measured in the Andreev quantum dot. However, the process $0 \leftrightarrow 2$ can occur too fast, on the frequencies exceeding the resolution of the single-electron transistors. In this case the two alternative recipes for measuring the excitation charge can be proposed. One is to measure the averaged equilibrium charge $Q_{\text {eq }}$ first at the temperature exceeding the level spacing and find $Q_{\mathrm{eq}}=Q_{1}$, then at temperatures below the the level spacing, $Q_{\mathrm{eq}}=Q_{0}$. The difference between the charges in these two experiments will give the excitation charge we look for $Q_{1}-Q_{0}$, see Eq. (52). As a result we get the fractional charge, depending continuously upon the external parameter $\varphi$. The second way to to measure the charge at low temperatures resolving the slow process $0 \leftrightarrow 1$ and averaging it with respect the fast $0 \leftrightarrow 2$, see Fig. 8 . The charge will be measured then in these two states, the state $|1\rangle$ and the state which is a thermodynamic equilibrium between $|0\rangle$ and $|2\rangle$. Let us calculate the difference between the average charges in these two states. The probabilities of filling states $|0\rangle,|1\rangle$, and $|2\rangle$ are equal to $p_{0}=\left[1-f_{\mathbb{T}}\left(\varepsilon_{\mathrm{A}}\right)\right]^{2}, p_{1}=2 f_{\mathbb{T}}\left(\varepsilon_{\mathrm{A}}\right)\left[1-f_{\mathbb{T}}\left(\varepsilon_{\mathrm{A}}\right)\right]$, and $p_{2}=f_{\mathbb{T}}^{2}\left(\varepsilon_{\mathrm{A}}\right)$, respectively. The averaging over the rapidly fluctuating regime including states $|0\rangle$ and $|1\rangle$, yields $\langle Q\rangle_{0,2}=p_{0} Q_{0}+p_{2} Q_{2}$, whereas the charge of a single excitation $\langle Q\rangle_{1}=Q_{1}$. Then at low temperatures, where $f_{\mathbb{T}}\left(\varepsilon_{\mathrm{A}}\right) \ll 1$, we recover again the excitation charge $\langle Q\rangle_{1}-\langle Q\rangle_{0,2}=Q_{\text {ex }}$.

\section{Measurement of the flux by the Andreev quantum dot}

In this section we will consider the dependence of the fractional charge of the Andreev quantum dot upon $\varphi$ in some more detail. This dependence can be very sharp, so that one can think of using the Andreev quantum dot for design of the new type of the magnetometer of measuring the low magnetic fields. Usually there are superconducting quantum interferometers, SQUIDs, that are used for that purpose [21, 44-46]. While the SQUIDs utilize the dependence of the Josephson current upon the difference of superconducting phases $\varphi$ (hence upon the magnetic magnetic flux $\Phi$ ), we propose to use the dependence of the charge of the Andreev quantum dot upon $\varphi$.

The charge of the Andreev quantum dot can be measured by the sensitive magnetometer, for example, by the single-electron transistor. The best transistors can resolve the charges of order $10^{-5}|e| / \mathrm{Hz}^{1 / 2}$ at given frequency, see, e.g. [43]. Simple estimates demonstrate that the change in the flux $\delta \Phi$ causes the variation of the charge $\delta Q$ as $\delta Q=\left(2|e| / \Phi_{0}\right) \delta \Phi$, where $\Phi_{0}=2 \pi \hbar / 2|e|$ is the flux quantum. Taking the area of the superconducting loop equal $1 \mathrm{~mm}^{2}$, we obtain the sensitivity $10^{-14} \mathrm{~T} / \mathrm{Hz}^{1 / 2}$, which favorably compares to the sensitivity of the best SQUIDs, $10^{-14} \div 10^{-15} \mathrm{~T} / \mathrm{Hz}^{1 / 2}$ [45-48]. Let us call the sensitivity of the Andreev quantum dot the ratio $\delta Q / \delta \Phi$.

The differential sensitivity of the charge in the thermodynamic equilibrium to the magnetic flux threading the superconducting ring we define as the modulus of the partial derivative $\partial Q_{\text {eq }} / \partial \Phi$, taken at given magnitude of the flux $\Phi: \mathcal{S}=\partial Q_{\text {eq }} / \partial \Phi$. Note that the sensitivity of the flux into the charge transformer described here, $\mathcal{S}=\mathcal{S}_{\Phi \rightarrow \mathrm{Q}}$, exactly coincides with the sensitivity of the Josephson transistor [8], which converts the gate potential into the current 
$\mathcal{S}_{\mathrm{V} \rightarrow \mathrm{I}}=\partial I_{\mathrm{eq}} / \partial V_{\mathrm{g}}$. Making use of the Eq. (53), we obtain

$$
\mathcal{S}=F_{\mathbb{T}} \frac{\partial Q}{\partial \Phi}+Q \frac{\partial F_{\mathbb{T}}}{\partial \Phi}
$$

where $Q=\left(Q_{+}-Q_{-}\right) / 2$, the derivative

$$
\frac{\partial Q}{\partial \Phi}=e \frac{2 \pi}{\Phi_{0}} \frac{\left(\varepsilon_{\mathrm{D}}+2 E_{\mathrm{C}}\right) \Gamma^{2} \sin \varphi}{4\left[\left(\varepsilon_{\mathrm{D}}+2 E_{\mathrm{C}}\right)^{2}+\tilde{\Gamma}^{2}\right]^{3 / 2}}
$$

defines the sensitivity of the charges of the states $|-\rangle$ and $|+\rangle$, and the function

$$
F_{\mathbb{T}}=\frac{e^{-E_{+} / k_{\mathrm{B}} \mathbb{T}}-e^{-E_{-} / k_{\mathrm{B}} \mathbb{T}}}{e^{-E_{-} / k_{\mathrm{B}} \mathbb{T}}+2 e^{-E_{1} / k_{\mathrm{B}} \mathbb{T}}+e^{-E_{+} / k_{\mathrm{B}} \mathbb{T}}} .
$$

describes the dependence of the charge upon the temperature and accounts for the the doublet region (50).

One can see from Fig. 11 there exist two intervals where the dependence of $Q_{\text {eq }}(\varphi)$ is sharp. If $\varphi$ increases from $\varphi=0$, the charge increases (decreases) and achieves the maximum (minimum). In case $E_{\mathrm{C}}<E_{\mathrm{C}}^{*}=\Gamma A$ the maximum (minimum) of the charge is always at the point $\varphi=\pi$. In this case the whole curve is the interval I. If $E_{\mathrm{C}}>E_{\mathrm{C}}^{*}$ this extremum splits into two and there appears a dent in between the extrema, which we will be calling the interval II. The former interval corresponds to a singlet state of the Andreev quantum dot, while the better part of the latter interval corresponds to a doublet state (in the case of the zero temperature the whole second interval corresponds to a doublet region).

Let us begin with the description of the first interval. Let us fix the parameters $\Gamma, A$ and $E_{\mathrm{C}}$ and seek for the maximum of the sensitivity $\mathcal{S}$ as function of $\varphi$ and $\varepsilon_{\mathrm{D}}$. The symmetries of the function $Q_{\mathrm{eq}}\left(\varphi, \varepsilon_{\mathrm{D}}\right)$ enable us to narrow the region of the search for maximum down to $0 \leqslant \varphi \leqslant \pi, \varepsilon_{\mathrm{D}}+2 E_{\mathrm{C}}>0$. After that we analyze maximum as function of $E_{\mathrm{C}}$, keeping $A$ and $\Gamma$ constant.

Interval $I$. In the case $E_{\mathrm{C}}<\sqrt{3} E_{\mathrm{C}}^{*}$ and zero temperature, $\mathbb{T}=0$, the sensitivity is completely defined by $\partial Q_{\text {ex }} / \partial \Phi(72)$. In the limit $A \ll 1$ the function $\left|\partial Q_{\text {ex }} / \partial \Phi\right|$ achieves the maximum at $\varepsilon_{\mathrm{D}}+2 E_{\mathrm{C}}=\Gamma \cos (\varphi / 2)=\Gamma A$, the maximum being

$$
\mathcal{S}_{\max }^{\mathrm{I}}=|e| \frac{2 \pi}{\Phi_{0}} \frac{1}{6 \sqrt{3} A} .
$$

One sees that the smaller $A$ imply larger sensitivity. In other words, more symmetric SINIS structure provides higher sensitivity. If $k_{\mathrm{B}} \mathbb{T} \ll E_{\mathrm{C}}^{*}$, the sensitivity is practically not temperature-dependent. 


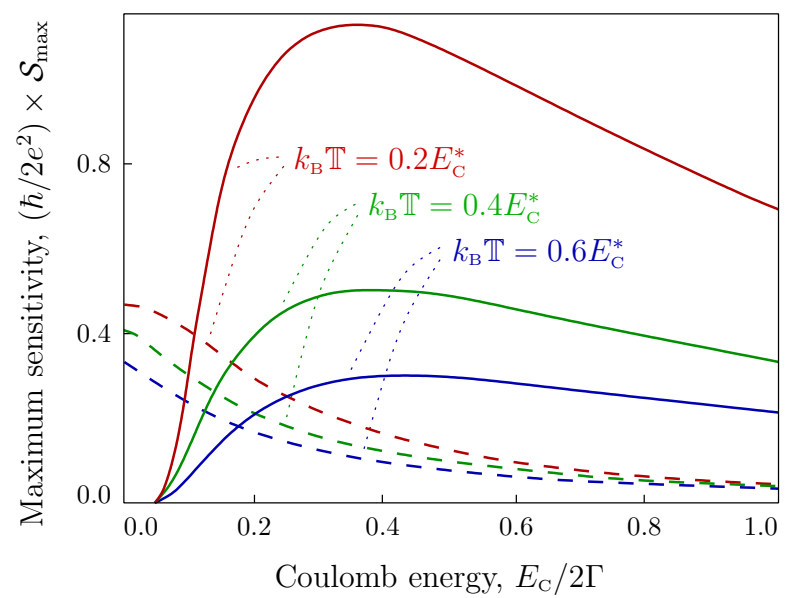

Figure 15. Maximal differential sensitivity in the interval I, $\mathcal{S}_{\max }^{\mathrm{I}}$ (the dashed line) and in the interval II, $\mathcal{S}_{\max }^{\mathrm{II}}$ (solid line) depending on the Coulomb energy $E_{\mathrm{C}}$. The level of asymmetry $A=0.2$; the corresponding critical Coulomb energy $E_{\mathrm{C}}^{*} / \Gamma=0.2$. The temperature varies from $k_{\mathrm{B}} \mathbb{T}=0.2 E_{\mathrm{C}}^{*}$ to $k_{\mathrm{B}} \mathbb{T}=0.6 E_{\mathrm{C}}^{*}$.

In the opposite case, $E_{\mathrm{C}}>\sqrt{3} E_{\mathrm{C}}^{*}$, the doublet region partially absorbs the interval $\mathrm{I}$ and maximum at zero temperature is achieved at the boundary of the doublet region. This yields the sensitivity

$$
\mathcal{S}_{\max }^{\mathrm{I}}=|e| \frac{2 \pi}{\Phi_{0}} \frac{\Gamma^{2}}{4 E_{\mathrm{C}}^{3}} \max _{\varphi}\left\{\sqrt{E_{\mathrm{C}}^{2}-\tilde{\Gamma}^{2}} \sin \varphi\right\} .
$$

The result is reduced to

$$
\mathcal{S}_{\text {max }}^{\mathrm{I}}=|e| \frac{2 \pi}{\Phi_{0}} \frac{\Gamma^{2}}{4 E_{\mathrm{C}}^{2}}
$$

in the limit $E_{\mathrm{C}} \gg \Gamma$. The maximum is achieved at the point $\varepsilon_{\mathrm{D}}+2 E_{\mathrm{C}}=E_{\mathrm{C}}, \varphi=\pi / 2$. Note that formula (76) gives the correct estimate even in the case $E_{\mathrm{C}} \approx \Gamma$.

Interval II. At zero temperature there is a jump in the charge at the boundary of the doublet region (interval II), therefore the sensitivity at this point is infinite. The finite temperature would smooth the jump, the sensitivity remaining finite. If $\Gamma \ll E_{\mathrm{C}} \ll \Gamma^{2} / k_{\mathrm{B}} \mathbb{T}$, the sensitivity $\mathcal{S}$ achieves its maximum near the point $\varepsilon_{\mathrm{D}}+2 E_{\mathrm{C}}=E_{\mathrm{C}}, \varphi=\pi / 2$ and is equal to

$$
\mathcal{S}_{\max }^{\mathrm{II}}=|e| \frac{2 \pi}{\Phi_{0}} \frac{\Gamma^{2}}{16 E_{\mathrm{C}} k_{\mathrm{B}} \mathrm{T}} .
$$

Note that this maximum is shifted at the finite temperature from the point of the phase transition towards the singlet over the quantity $\delta \varphi \sim E_{\mathrm{C}} k_{\mathrm{B}} \mathbb{T} / 4 \Gamma^{2}$. For the arbitrary $E_{\mathrm{C}}$ the expression for the $\mathcal{S}_{\max }^{\mathrm{II}}$ is somewhat cumbersome. We have shown the numerically found dependence $\mathcal{S}_{\max }^{\mathrm{II}}\left(E_{\mathrm{C}}\right)$ in Fig. 15 , where we also presented the maximum of sensitivity in the 
interval I. One sees that under the large Coulomb interaction the better sensitivity is achieved in the interval II.

Therefore the dependence of the charge of the Andreev quantum dot upon the the difference of the superconducting phases can be used, in principle, for the design of the new type of the of the quantum magnetometers working according to the scheme "magnetic flux $\rightarrow$ charge of the Andreev dot $\rightarrow$ charge detector $\rightarrow$ current" instead of the usual scheme of customary SQUIDs "magnetic flux $\rightarrow$ current."

Let us estimate the sensitivity of the Andreev quantum point-based magnetometer, comparing them to the existing and widely used SQUIDs that can resolve at best the flux $10^{-6} \Phi_{0} / \mathrm{Hz}^{1 / 2}$ at given frequency. The typical SQUID area is $2 \mathrm{~mm}^{2}$, implying the resolution of the magnetic field $10^{-15} \mathrm{~T} / \mathrm{Hz}^{1 / 2}$ at given frequency. Assume the area of the superconducting ring the same as in SQUIDs and take the resolution of the charge detector equal to $10^{-5}|e| / \mathrm{Hz}^{1 / 2}$ [43]. In the limit of the small Coulomb interaction, the sensitivity is determined by the asymmetry of the quantum dot. At $A=0.01$ Eq. (74) gives for the flux $2 \times 10^{-7} \Phi_{0} / \mathrm{Hz}^{1 / 2}$ and for the field $2 \times 10^{-16} \mathrm{~T} / \mathrm{Hz}^{1 / 2}$. Upon increase of the Coulomb interaction the maximal sensitivity is located at the boundary of the doublet region and is determined by the magnitude of the Coulomb energy. Plugging in $E_{\mathrm{C}}=6 \Gamma$, and using Eq. (76), we find the flux resolution $3 \times 10^{-4} \Phi_{0} / \mathrm{Hz}^{1 / 2}$ and the field resolution $3 \times 10^{-13} \mathrm{~T} / \mathrm{Hz}^{1 / 2}$. The sensitivity that can be achieved upon transition from the singlet into the doublet region is restricted from above by the temperature of the measurements. Plugging in $k_{\mathrm{B}} \mathrm{T}=0.02 \Gamma$ and $E_{\mathrm{C}}=0.2 \Gamma$ into Eq. (77), we find for the flux $10^{-7} \Phi_{0} / \mathrm{Hz}^{1 / 2}$ and for the field $10^{-16} \mathrm{~T} / \mathrm{Hz}^{1 / 2}$.

In real devices the Coulomb interaction $E_{\mathrm{C}}$ can be less than $\Gamma$ (see discussion in Refs. [10, 27]) or larger than $\Gamma$ (see Refs. $[28,29])$. The second case can be realized, for example, raising the Fermi level in the normal region. Nanomechanical effects also can either suppress or enhance the sensitivity of the Andreev quantum dot with respect to the phase difference [49].

\section{Conclusions}

We discussed in depth the charge of the Andreev quantum dot. We demonstrated that the charge is localized in the region of the dot and depends continuously upon the difference of the phases between the superconductors. We studied charge fluctuations due to both, electron-phonon interactions and due to fluctuations of the gate potential. We revealed that Coulomb interaction can cause the reconstruction of the singlet ground state in the doublet one. While in the singlet state the Josephson current is present and the charge does depend on the phase, in the doublet state the non-dissipative current is absent and the charge is integer. Upon increasing the charge energy, the "size" of the doublet region in the $\left(\varphi, \varepsilon_{\mathrm{D}}\right)$ coordinates increases as well.

We discussed a concept of a novel device for measurements of the magnetic flux, utilizing the Andreev quantum dot as a working element, and investigated its differential sensitivity. The estimates show that the expected theoretical sensitivity of the Andreev quantum dot magnetometer compares favorably with the characteristics of the existing SQUIDs. 


\section{Acknowledgements}

We thank G. Blatter, T. Martin, T. Jonckheere, N.B. Kopnin, and V.V. Ryazanov for illuminating discussions. The work was supported by the Scientific Discovery through Advanced Computing (SciDAC) program funded by U.S. Department of Energy, Office of Science, Advanced Scientific Computing Research and Basic Energy Science (I.S.), U.S. Department of Energy, Office of Science, Materials Sciences and Engineering Division (V.V. and, partly, G.L. via Materials Theory Institute), and by the RFBR Grant No. 14-02-01287 (G.L.).

\section{Author details}

Ivan A. Sadovskyy ${ }^{1}$, Gordey B. Lesovik ${ }^{2}$, Valerii M. Vinokur ${ }^{1}$

1 Materials Science Division, Argonne National Laboratory, Argonne, Illinois, USA

2 Landau Institute for Theoretical Physics, RAS, Chernogolovka, Moscow region, Russia

\section{References}

[1] B.D. Josephson. Possible new effects in superconductive tunnelling. Phys. Lett., 1: 251-253, 1962.

[2] P.G. de Gennes. Boundary effects in superconductors. Rev. Mod. Phys., 36(1):225-237, Jan 1964.

[3] A.A. Abrikosov. On the magnetic properties of superconductors of the second group. Sov. Phys. JETP, 5(6):1174-1182, Dec 1957.

[4] D.I. Khomskii and A. Freimuth. Charged vortices in high temperature superconductors. Phys. Rev. Lett., 75(7):1384-1386, Aug 1995.

[5] G. Blatter, M. Feigel'man, V. Geshkenbein, A. Larkin, and A. van Otterlo. Electrostatics of vortices in type-II superconductors. Phys. Rev. Lett., 77(3):566-569, Jul 1996.

[6] H. van Houten. Three-terminal quantum box resonant tunneling Josephson field-effect switch. Appl. Phys. Lett., 58(12):1326-1328, 1991.

[7] G. Wendin and V.S. Shumeiko. Josephson transport in complex mesoscopic structures. Superlatt. and Microstruct., 20(4):569-573, Dec 1996.

[8] D.D. Kuhn, N.M. Chtchelkatchev, G.B. Lesovik, and G. Blatter. Supercurrents through gated superconductor-normal-metal-superconductor contacts: The Josephson transistor. Phys. Rev. B, 63(5):054520, Jan 2001.

[9] N.M. Chtchelkatchev and Yu.V. Nazarov. Andreev quantum dots for spin manipulation. Phys. Rev. Lett., 90(22):226806, Jun 2003.

[10] I.A. Sadovskyy, G.B. Lesovik, and G. Blatter. Continuously tunable charge in Andreev quantum dots. Phys. Rev. B, 75(19):195334, May 2007. 
[11] K. Engström and J. Kinaret. Phase-dependent charges in SNS systems. Phys. Scr., 70(5): 326-329, 2004.

[12] R.B. Laughlin. Anomalous quantum Hall effect: an incompressible quantum fluid with fractionally charged excitations. Phys. Rev. Lett., 50(18):1395-1398, May 1983.

[13] L. Saminadayar, D.C. Glattli, Y. Jin, and B. Etienne. Observation of the $e / 3$ fractionally charged Laughlin quasiparticle. Phys. Rev. Lett., 79(13):2526-2529, Sep 1997.

[14] M. Reznikov, R. de Picciotto, T.G. Griffiths, M. Heiblum, and V. Umansky. Observation of quasiparticles with one-fifth of an electron's charge. Nature, 399(6733):238-241, May 1999.

[15] X.G. Wen. Gapless boundary excitations in the quantum Hall states and in the chiral spin states. Phys. Rev. B, 43(13):11025-11036, May 1991.

[16] A.V. Lebedev, A. Crépieux, and T. Martin. Electron injection in a nanotube with leads: finite-frequency noise correlations and anomalous charges. Phys. Rev. B, 71(7):075416, Feb 2005.

[17] B. Trauzettel, I. Safi, F. Dolcini, and H. Grabert. Fractional charge in the noise of Luttinger liquid systems. In Peter Svedlindh, Dragana Popovic, and Michael B. Weissman, editors, Fluctuations and noise in materials II, volume 5843, pages 115-123. SPIE, 2005.

[18] M. Büttiker and C.A. Stafford. Charge transfer induced persistent current and capacitance oscillations. Phys. Rev. Lett., 76(3):495-498, Jan 1996.

[19] P.S. Deo, P. Koskinen, and M. Manninen. Charge fluctuations in coupled systems: ring coupled to a wire or ring. Phys. Rev. B, 72(15):155332, Oct 2005.

[20] C.J. Pethick and H. Smith. Relaxation and collective motion in superconductors: a two-fluid description. Annals of Physics, 119(1):133-169, 1979. ISSN 0003-4916. .

[21] V.V. Schmidt. The physics of superconductors: Introduction to fundamentals and applications. Springer, 2002.

[22] I.A. Sadovskyy, G.B. Lesovik, and G. Blatter. Magnetic flux detection with an Andreev quantum dot. JETP Lett., 86:210, 2007.

[23] A.F. Andreev. Thermal conductivity of the intermediate state of superconductors. Sov. Phys. JETP, 19:1228-1231, 1964.

[24] A.F. Andreev. Thermal conductivity of the intermediate state of superconductors - II. Sov. Phys. JETP, 20:1490-1493, 1965.

[25] A.F. Andreev. Electron spectrum of the intermediate state of superconductors. Sov. Phys. JETP, 22:455-458, 1966.

[26] M.R. Buitelaar, T. Nussbaumer, and C. Schönenberger. Quantum dot in the Kondo regime coupled to superconductors. Phys. Rev. Lett., 89(25):256801, Dec 2002. 
[27] P. Jarillo-Herrero, J.A. van Dam, and L.P. Kouwenhoven. Quantum supercurrent transistors in carbon nanotubes. Nature, 439:953-956, Feb 2006.

[28] J.A. van Dam, Yu.V. Nazarov, E.P.A.M. Bakkers, S. De Franceschi, and L.P. Kouwenhoven. Supercurrent reversal in quantum dots. Nature, 442:667-670, Aug 2006.

[29] J.-P. Cleuziou, W. Wernsdorfer, V. Bouchiat, T. Ondarọuhu, and M. Monthioux. Carbon nanotube superconducting quantum interference device. Nature Nanotech., 1:53-59, Oct 2006.

[30] I.A. Sadovskyy, G.B. Lesovik, G. Blatter, T. Jonckheere, and T. Martin. Andreev quantum dot with several conducting channels. Phys. Rev. B, 85:125442, Mar 2012.

[31] N. Roch, S. Florens, V. Bouchiat, W. Wernsdorfer, and F. Balestro. Quantum phase transition in a single-molecule quantum dot. Nature, 453(7195):633-637, 052008.

[32] C.B. Winkelmann, N. Roch, W. Wernsdorfer, V. Bouchiat, and F. Balestro. Superconductivity in a single- $\mathrm{C}_{60}$ transistor. Nat. Phys., 5(12):876-879, 122009.

[33] I.A. Sadovskyy, D. Chevallier, T. Jonckheere, M. Lee, S. Kawabata, and T. Martin. Josephson effect through an anisotropic magnetic molecule. Phys. Rev. B, 84:184513, Nov 2011.

[34] G.B. Lesovik and I.A. Sadovskyy. Scattering matrix approach to the description of quantum electron transport. Physics-Uspekhi, 54(10):1007-1059, 2011.

[35] N.M. Chtchelkatchev, G.B. Lesovik, and G. Blatter. Supercurrent quantization in narrow-channel superconductor-normal-metal-superconductor junctions. Phys. Rev. B, 62(5):3559-3564, Aug 2000.

[36] D.A. Ivanov and M.V. Feigel'man. Phonon relaxation of subgap levels in superconducting quantum point contacts. JETP Lett., 68(11):890-894, Dec 1998.

[37] A.V. Rozhkov and D.P. Arovas. Interacting-impurity Josephson junction: variational wave functions and slave-boson mean-field theory. Phys. Rev. B, 62(10):6687-6691, Sep 2000.

[38] H.B. Heersche, P. Jarillo-Herrero, J.B. Oostinga, L.M.K. Vandersypen, and A.F. Morpurgo. Bipolar supercurrent in graphene. Nature, 446:56-59, Mar 2007.

[39] V.I. Fal'ko and G.B. Lesovik. Quantum conductance fluctuations in 3D ballistic adiabatic wires. Solid State Comm., 84(8):835-837, 1992.

[40] M. Titov and C.W.J. Beenakker. Josephson effect in ballistic graphene. Phys. Rev. B, 74 (4):041401, Jul 2006.

[41] J.C. Cuevas and A. Levy Yeyati. Subharmonic gap structure in short ballistic graphene junctions. Phys. Rev. B, 74(18):180501, Nov 2006.

[42] C.W.J. Beenakker. Specular Andreev reflection in graphene. Phys. Rev. Lett., 97(6):067007, Aug 2006. 
[43] A. Aassime, G. Johansson, G. Wendin, R.J. Schoelkopf, and P. Delsing. Radio-frequency single-electron transistor as readout device for qubits: charge sensitivity and backaction. Phys. Rev. Lett., 86(15):3376-3379, Apr 2001.

[44] J. Clarke. SQUIDs for low frequency measurements. In Brian B. Schwartz and Simon Foner, editors, Superconductor applications: SQUIDs and machines, pages 67-124, New York, 1977. Plenum Press.

[45] J. Clarke and A.I. Braginski, editors. Fundamentals and technology of SQUIDs and SQUID systems, volume 1 of The SQUID Handbook. Wiley-VCH, Berlin, Germany, 2004.

[46] J. Clarke and A.I. Braginski, editors. Applications of SQUIDs and SQUID systems, volume 2 of The SQUID Handbook. Wiley-VCH, Berlin, Germany, 2004.

[47] R. Kleiner, D. Koelle, F. Ludwig, and J. Clarke. Electrostatics of vortices in type-II superconductors. Proc. of the IEEE, 92(10):1534-1548, Oct 2004.

[48] H. Weinstock, editor. Applications of Superconductivity, volume 365 of Series E: Applied Sciences. Kluwer, Dordrecht, The Netherlands, 2000.

[49] I.A. Sadovskyy, G.B. Lesovik, T. Jonckheere, and T. Martin. Nanomechanical effects in an Andreev quantum dot. Phys. Rev. B, 82:235310, Dec 2010. 
